\title{
An error indicator for mortar element solutions to the Stokes problem
}

\author{
CHRISTINE BERNARDi \\ Laboratoire d'Analyse Numérique, CNRS \& Université Pierre et Marie Curie, \\ BC 187, 4 Place Jussieu, 75252 Paris Cedex 05, France \\ NiCOLAS FiÉTIER \\ DGM - IMHEF - LIN, École Polytechnique Fédérale de Lausanne, CH 1015 \\ Lausanne, Switzerland \\ AND \\ ROBERT G. OWENS \\ DGM - IMHEF - LMF, École Polytechnique Fédérale de Lausanne, CH 1015 \\ Lausanne, Switzerland
}

[Received on 6 September 1999; revised on 8 May 2001; accepted on 11 May 2001]

\begin{abstract}
We are interested in the mortar spectral element discretization of the Stokes problem in a two-dimensional polygonal domain. We propose a family of residual type error indicators and we prove estimates which allow us to compare them with the error. We explain how these indicators can be used for adaptivity, and we present some numerical experiments.
\end{abstract}

Keywords: mortar element method; error indicator; Stokes problem; mesh adaptivity.

\section{Introduction}

The mortar element method (Bernardi et al., 1994; Maday et al., 1989) has proved useful in extending the flexibility of the spectral element approach by providing a framework within which non-conforming discretizations are possible. The mortar element method is of particular value in coupling spectral and finite element methods (Bernardi et al., 1994) and in adaptive applications (Azaïez et al., 1996). Recent work has seen the method applied to fourth-order problems (Belhachmi, 1997; Belhachmi \& Bernardi, 1994), to wavelets (Bertoluzza \& Perrier, 0000, 2001) and to overlapping sub-domains (Cai et al., 1997). In the context of the Stokes problem, Babuška-Brezzi type conditions have now been proved for fixed mortar element decompositions and a finite hierarchical family of mortar element decompositions (Ben Belgacem et al., 2000).

In this paper we seek, for the first time, to derive in detail an a posteriori error indicator for mortar element solutions to the Stokes problem. Our inspiration comes from earlier work on residual based error indicators derived by Verfürth (1989, 1991, 1994) and Bernardi et al. (0000) for finite element solutions to the Stokes problem. A comparative study by Bank \& Welfert (1990) of three different error indicators for the Stokes problem in various two-dimensional geometries found that a version of Verfürth's indicator (Verfürth, 1989) involving the solution of local Stokes problems gave a more 
accurate indication of the global error than a variation on this indicator based on local residual evaluations. However, the computational cost of the latter was less than half that of the other. Moreover, the residual evaluation indicator is comparatively straightforward to extend to non-conforming discretizations and has been seen to be adequate for adaptive spectral element methods (Azaïez et al., 1996). We also refer to Wohlmuth (1999) and Bernardi \& Hecht (0000) for first studies of such indicators in the finite element mortar framework. Of course, if one is prepared to pay the computational cost there exist reliable error estimators of the type derived by Ainsworth \& Oden (1997) for the Stokes and Oseen equations, for example, involving the solution of residual problems and the use of a flux equilibration procedure. However, this estimator has been derived for continuous finite element approximations.

The present work is an extension of the indicator for spectral elements derived by Bernardi (1996) for model one- and two-dimensional elliptic problems. This latter reference concluded by only briefly outlining a procedure whereby error indicators could be established for mortar element solutions to Poisson's problem. As an extension of the results of Bernardi (1996) we propose a family of error indicators for mortar element solutions to the two-dimensional Stokes problem and we perform the corresponding $a$ priori analysis. We describe the implementation of the adaptivity algorithm, together with some numerical experiments which are in good agreement with the results of the analysis.

The outline of the paper is as follows. In Section 2 we describe the Stokes problem in a two-dimensional domain and write down its discretization by combining spectral approximations on each subdomain via the mortar element technique. In Section 3 we propose an error indicator and prove in detail the estimates that allow us to compare the indicator with the error in the energy norm. The key results are stated in Theorems 1 and 2. Finally, in Section 4, we explain how mesh adaptivity can be performed and we present the results of some numerical experiments.

\section{The two-dimensional Stokes problem}

Let $\Omega$ be a bounded open set in $\mathbb{R}^{2}$ with a Lipschitz-continuous boundary $\partial \Omega$ and generic point $(x, y)$. We first describe the main properties of the Stokes problem in this domain. Next, we present its mortar spectral element discretization when the domain $\Omega$ admits a disjoint partition into quadrilaterals.

\subsection{Problem description}

We consider the Stokes problem

$$
\begin{aligned}
-v \nabla^{2} \mathbf{u}+\nabla p=\mathbf{f}, & \text { in } \Omega, \\
\nabla \cdot \mathbf{u}=0, & \text { in } \Omega, \\
\mathbf{u}=\mathbf{0}, & \text { on } \partial \Omega,
\end{aligned}
$$

where the positive constant $v$ represents the viscosity, $\mathbf{u}=(u, v)$ is the fluid velocity, $p$ the pressure and $\mathbf{f}$ a body force. 
The variational formulation of this problem relies on the standard Sobolev spaces $H^{m}(\Omega)$, provided with the usual norms and seminorms. We define

$$
X(\Omega)=H_{0}^{1}(\Omega)^{2},
$$

next we introduce the space

$$
L_{0}^{2}(\Omega)=\left\{q \in L^{2}(\Omega) ; \int_{\Omega} q(x, y) \mathrm{d} x \mathrm{~d} y=0\right\},
$$

and then denote by $Z(\Omega)$ the space $X(\Omega) \times L_{0}^{2}(\Omega)$. Since functions in $X(\Omega)$ vanish on $\partial \Omega$, a norm $\|\cdot\|_{Z}$ may be defined on $Z(\Omega)$ by

$$
\|(\mathbf{u}, p)\|_{Z}=|\mathbf{u}|_{H^{1}(\Omega)^{2}}+\|p\|_{L^{2}(\Omega)} .
$$

We now introduce a bilinear form $\mathcal{L}: Z(\Omega) \times Z(\Omega) \longrightarrow \mathbb{R}$ defined for all $(\mathbf{u}, p)$ and $(\mathbf{v}, q)$ in $Z(\Omega)$ by

$$
\mathcal{L}((\mathbf{u}, p) ;(\mathbf{v}, q))=v \int_{\Omega}(\nabla \mathbf{u})^{T}:(\nabla \mathbf{v}) \mathrm{d} x \mathrm{~d} y-\int_{\Omega} p \nabla \cdot \mathbf{v} \mathrm{d} x \mathrm{~d} y-\int_{\Omega} \nabla \cdot \mathbf{u} q \mathrm{~d} x \mathrm{~d} y .
$$

Then a weak statement of problem (1)-(3) is: find $(\mathbf{u}, p)$ in $Z(\Omega)$ such that

$$
\forall(\mathbf{v}, q) \in Z(\Omega), \quad \mathcal{L}((\mathbf{u}, p) ;(\mathbf{v}, q))=\int_{\Omega} \mathbf{f} \cdot \mathbf{v} \mathrm{d} x \mathrm{~d} y .
$$

If we define the bilinear forms

$$
a: X(\Omega) \times X(\Omega) \longrightarrow \mathbb{R}, \quad b: X(\Omega) \times L_{0}^{2}(\Omega) \longrightarrow \mathbb{R}
$$

by

$$
\begin{aligned}
& a(\mathbf{u}, \mathbf{v})=v \int_{\Omega}(\nabla \mathbf{u})^{T}:(\nabla \mathbf{v}) \mathrm{d} x \mathrm{~d} y, \\
& b(\mathbf{v}, p)=-\int_{\Omega} p \nabla \cdot \mathbf{v} \mathrm{d} x \mathrm{~d} y,
\end{aligned}
$$

then we can write

$$
\mathcal{L}((\mathbf{u}, p) ;(\mathbf{v}, q))=a(\mathbf{u}, \mathbf{v})+b(\mathbf{v}, p)+b(\mathbf{u}, q) .
$$

It follows, as a consequence of the ellipticity of $a(\cdot, \cdot)$ and the inf-sup condition (Brezzi, 1974, Girault \& Raviart, 1986, Chapter I, Corollary 2.4)

$$
\forall p \in L_{0}^{2}(\Omega), \quad \operatorname{Sup}_{\mathbf{v} \in X(\Omega)} \frac{b(\mathbf{v}, p)}{|\mathbf{v}|_{H^{1}(\Omega)^{2}}} \geqslant \beta_{1}\|p\|_{L^{2}(\Omega)},
$$

for some positive number $\beta_{1}$, that for any data $\mathbf{f}$ in $H^{-1}(\Omega)^{2}$, problem (4) has a unique solution. Moreover, the mapping: $(\mathbf{f}, g) \mapsto(\mathbf{u}, p)$, where $(\mathbf{u}, p)$ is the solution of problem (1)-(3) with the equation $\nabla \cdot \mathbf{u}=0$ replaced by $\nabla \cdot \mathbf{u}=g$, is an isomorphism 
from $H^{-1}(\Omega)^{2} \times L_{0}^{2}(\Omega)$ onto $H_{0}^{1}(\Omega)^{2} \times L^{2}(\Omega) / \mathbb{R}$. This yields (Girault \& Raviart, 1986, Chapter I, Lemma 4.1) the further condition

$$
\forall(\mathbf{u}, p) \in Z(\Omega), \quad \operatorname{Sup}_{(\mathbf{v}, q) \in Z(\Omega)} \frac{\mathcal{L}((\mathbf{u}, p) ;(\mathbf{v}, q))}{\|(\mathbf{v}, q)\|_{Z}} \geqslant \beta_{2}\|(\mathbf{u}, p)\|_{Z},
$$

where $\beta_{2}$ is some positive number.

In view of the discretization and also of the adaptive strategy, we also recall the regularity properties of the solution of problem (1)-(3). Indeed, even for smooth data, it is limited by the apparition of some singular functions linked to the corners of the domain (Bernardi \& Raugel, 1981; Osborn, 1976). So, for data $\mathbf{f}$ in $H^{s-2}(\Omega)$, the solution $(\mathbf{u}, p)$ belongs to $H^{s}(\Omega)^{2} \times H^{s-1}(\Omega)$ for all $s \leqslant 2$ when $\Omega$ is convex and for all $s \leqslant 1+\eta(\omega)$ when $\Omega$ is a polygon with largest angle $\omega$, with

$$
\eta\left(\frac{\pi}{2}\right) \simeq 2.739 \text { and } \eta\left(\frac{3 \pi}{2}\right) \simeq 0.544 .
$$

However, the support of these singular functions is local so that the regularity is limited only in a neighbourhood of the corners.

\subsection{Mortar element discretisation}

We now assume that $\Omega$ admits a decomposition into (say) $K$ quadrilateral elements $\left\{\Omega_{k}\right\}_{k=1}^{K}$ :

$$
\bar{\Omega}=\bigcup_{k=1}^{K} \bar{\Omega}_{k} \quad \text { and } \quad \Omega_{k} \cap \Omega_{k^{\prime}}=\emptyset, \quad 1 \leqslant k<k^{\prime} \leqslant K .
$$

We only make the further assumption that the intersection of each $\partial \Omega_{k}$ with $\partial \Omega$, if not empty, is made of corners or whole edges of $\Omega_{k}$.

For $1 \leqslant k \leqslant K$, we denote by $\Gamma_{k, l}, 1 \leqslant l \leqslant L(k)$, the edges of $\Omega_{k}$ that are not contained in $\partial \Omega$. We introduce the skeleton $\mathcal{S}$ of the decomposition:

$$
\mathcal{S}=\bigcup_{k=1}^{K} \partial \Omega_{k} \backslash \partial \Omega=\bigcup_{k=1}^{K} \bigcup_{l=1}^{L(k)} \Gamma_{k, l},
$$

and we choose a fixed disjoint decomposition of this skeleton into mortars:

$$
\mathcal{S}=\bigcup_{m=1}^{M} \gamma_{m} \quad \text { and } \quad \gamma_{m} \cap \gamma_{m^{\prime}}=\emptyset, \quad 1 \leqslant m<m^{\prime} \leqslant M,
$$

where each $\gamma_{m}$ is a whole edge $\Gamma_{k(m), l(m)}$ of a subdomain $\Omega_{k}$, here denoted by $\Omega_{k(m)}$. We also define the set $\mathcal{V}$ of all the corners of the subdomains $\Omega_{k}$.

For any non-negative integer $n$, let $\mathbb{P}_{n}\left(\Omega_{k}\right)$ denote the space of restrictions to $\Omega_{k}$ of all polynomials with degree $\leqslant n$ with respect to each variable $x$ and $y$. The discretization parameter is a $K$-tuple $N$ of integers $N_{k} \geqslant 2,1 \leqslant k \leqslant K$. We define our discrete spectral approximation space in the standard mortar fashion (Bernardi et al., 1994; Maday et al., 1989): it is the space $U_{N}\left(\cup \Omega_{k}\right)$ of functions $u_{N}$ such that 
1. each $\left.u_{N}\right|_{\Omega_{k}}, 1 \leqslant k \leqslant K$, belongs to $\mathbb{P}_{N_{k}}\left(\Omega_{k}\right)$,

2. $u_{N}$ vanishes on $\partial \Omega$,

3. the following matching condition holds on any edge $\Gamma_{k, l}, 1 \leqslant k \leqslant K, 1 \leqslant l \leqslant L(k)$,

$$
\forall \psi \in \mathbb{P}_{N_{k}-2}\left(\Gamma_{k, l}\right), \int_{\Gamma_{k, l}}\left(\left.u_{N}\right|_{\Omega_{k}}-\phi\right)(\tau) \psi(\tau) \mathrm{d} \tau=0,
$$

where the mortar function $\phi$ is defined on the skeleton $\mathcal{S}$ by

$$
\left.\phi\right|_{\gamma^{m}}=\left.\left.u_{N}\right|_{\Omega_{k(m)}}\right|_{\gamma^{m}}, \quad 1 \leqslant m \leqslant M .
$$

The discrete non-conforming velocity space $X_{N}$ is then defined by

$$
X_{N}=U_{N}\left(\cup \Omega_{k}\right) \times U_{N}\left(\cup \Omega_{k}\right) .
$$

We propose two different choices for the discrete pressure space, which we denote by $M_{N}^{1}$ and $M_{N}^{2}$. The first one is defined by

$$
M_{N}^{1}=\left\{q_{N} \in L_{0}^{2}(\Omega):\left.q_{N}\right|_{\Omega_{k}} \in \mathbb{P}_{N_{k}-2}\left(\Omega_{k}\right)\right\} .
$$

However, a second space, suggested in Bernardi \& Maday (1999), leads to a better a priori error estimate on the pressure: for a real number $\lambda, 0<\lambda<1$,

$$
M_{N}^{2}=\left\{q_{N} \in L_{0}^{2}(\Omega):\left.q_{N}\right|_{\Omega_{k}} \in \mathbb{P}_{N_{k}-2}\left(\Omega_{k}\right) \cap \mathbb{P}_{\left[\lambda N_{k}\right]}\left(\Omega_{k}\right)\right\},
$$

where $\left[\lambda N_{k}\right]$ stands for the integral part of $\lambda N_{k}$.

In order to handle discontinuous functions, we introduce the 'broken' norm and seminorm defined by

$$
\|v\|_{H^{1}\left(\cup \Omega_{k}\right)}=\left(\sum_{k=1}^{K}\|v\|_{H^{1}\left(\Omega_{k}\right)}^{2}\right)^{\frac{1}{2}}, \quad|v|_{H^{1}\left(\cup \Omega_{k}\right)}=\left(\sum_{k=1}^{K}|v|_{H^{1}\left(\Omega_{k}\right)}^{2}\right)^{\frac{1}{2}},
$$

and we extend the form $b(\cdot, \cdot)$ defined in (6) as follows:

$$
b(\mathbf{v}, q)=-\sum_{k=1}^{K} \int_{\Omega_{k}} q \nabla \cdot \mathbf{v} \mathrm{d} x \mathrm{~d} y .
$$

Both choices of the discrete pressure spaces ensure that the approximation spaces for the velocity components and pressure are compatible (Ben Belgacem et al., 2000; Maday et al., 1992). Indeed, the following inf-sup condition holds for $m=1$ and 2: there exists an integer $N_{D}$ depending only on the decomposition such that, if all the $N_{k}$ are $\geqslant N_{D}$, $1 \leqslant k \leqslant K$, then

$$
\forall q_{N} \in M_{N}^{m}, \quad \operatorname{Sup}_{\mathbf{v}_{N} \in X_{N}} \frac{b\left(\mathbf{v}_{N}, q_{N}\right)}{\left\|\mathbf{v}_{N}\right\|_{H^{1}\left(\cup \Omega_{k}\right)^{2}}} \geqslant \beta_{N}^{m}\left\|q_{N}\right\|_{L^{2}(\Omega)},
$$

for some positive number $\beta_{N}^{m}$ such that: 
- for the space $M_{N}^{1}$, the constant $\beta_{N}^{1}$ is $\geqslant \beta_{3}\left(\sup _{1 \leqslant k \leqslant K} N_{k}\right)^{-\frac{1}{2}}$,

- for the space $M_{N}^{2}$, the constant $\beta_{N}^{2}$ is $\geqslant \beta_{3}$,

where in both cases the constant $\beta_{3}$ only depends on the decomposition into subdomains.

Let us denote by $\left(x_{i}^{k}, y_{j}^{k}\right), 0 \leqslant i, j \leqslant N_{k}$, the point in $\Omega_{k}$ under the inverse mapping of the $(i, j)$ th Gauss-Lobatto point in the parent element $\widehat{\Omega}=[-1,1]^{2}$. If $\mathcal{J}_{k}$ denotes the Jacobian of the mapping from $\Omega_{k}$ to $\widehat{\Omega}$ and $\rho_{i}^{k}$ the $i$ th quadrature weight of the standard Gauss-Lobatto formula, we may define a discrete product in $\Omega_{k}$ by

$$
(u, v)_{N}^{k}=\sum_{i=0}^{N_{k}} \sum_{j=0}^{N_{k}} u\left(x_{i}^{k}, y_{j}^{k}\right) v\left(x_{i}^{k}, y_{j}^{k}\right) \mathcal{J}_{k}(i, j) \rho_{i}^{k} \rho_{j}^{k},
$$

and note that this coincides with the scalar product of $L^{2}\left(\Omega_{k}\right)$ whenever the product $u v \mathcal{J}_{k}$ belongs to $\mathbb{P}_{2 N_{k}-1}\left(\Omega_{k}\right)$. We are in a position to define the discrete bilinear form

$$
a_{N}\left(\mathbf{u}_{N}, \mathbf{v}_{N}\right)=\sum_{k=1}^{K}\left(\nabla \mathbf{u}_{N}, \nabla \mathbf{v}_{N}\right)_{N}^{k}
$$

As proven in Ben Belgacem et al. (2000, Theorem A.2), this form satisfies the following ellipticity property:

$$
\forall \mathbf{u}_{N} \in X_{N}, \quad a_{N}\left(\mathbf{u}_{N}, \mathbf{u}_{N}\right) \geqslant \alpha\left\|\mathbf{u}_{N}\right\|_{H^{1}\left(\cup \Omega_{k}\right)^{2}}^{2},
$$

where the constant $\alpha$ only depends on the diameter of $\Omega$ and the decomposition into subdomains.

Let us introduce the notation $Z_{N}^{m}$ for the space $X_{N} \times M_{N}^{m}$. In the obvious way, the norm $\|\cdot\|_{Z}$ is extended to piecewise continuous functions by

$$
\|(\mathbf{u}, p)\|_{Z}=|\mathbf{u}|_{H^{1}\left(\cup \Omega_{k}\right)^{2}}+\|p\|_{L^{2}(\Omega)} .
$$

Moreover, if we define the bilinear form $\mathcal{L}_{N}(\cdot, \cdot)$ by

$$
\mathcal{L}_{N}\left(\left(\mathbf{u}_{N}, p_{N}\right) ;\left(\mathbf{v}_{N}, q_{N}\right)\right)=a_{N}\left(\mathbf{u}_{N}, \mathbf{v}_{N}\right)+b\left(\mathbf{v}_{N}, p_{N}\right)+b\left(\mathbf{u}_{N}, q_{N}\right),
$$

then it follows that as a consequence of the ellipticity property (20) of the bilinear form $a_{N}(\cdot, \cdot)$ and the inf-sup condition (17) on $b(\cdot, \cdot)$ as defined by (16) that there exists some positive number $\tilde{\beta}_{N}^{m}$, possibly depending on $N$, such that

$$
\forall\left(\mathbf{u}_{N}, p_{N}\right) \in Z_{N}^{m}, \quad \operatorname{Sup}_{\left(\mathbf{v}_{N}, q_{N}\right) \in Z_{N}^{m}} \frac{\mathcal{L}_{N}\left(\left(\mathbf{u}_{N}, p_{N}\right) ;\left(\mathbf{v}_{N}, q_{N}\right)\right)}{\left\|\left(\mathbf{v}_{N}, q_{N}\right)\right\|_{Z}} \geqslant \tilde{\beta}_{N}^{m}\left\|\left(\mathbf{u}_{N}, p_{N}\right)\right\|_{Z} .
$$

With this notation, the discrete problem may now be written: find $\left(\mathbf{u}_{N}, p_{N}\right)$ in $Z_{N}^{m}$ such that

$$
\forall\left(\mathbf{v}_{N}, q_{N}\right) \in Z_{N}^{m}, \quad \mathcal{L}_{N}\left(\left(\mathbf{u}_{N}, p_{N}\right) ;\left(\mathbf{v}_{N}, q_{N}\right)\right)=\sum_{k=1}^{K}\left(\mathbf{f}, \mathbf{v}_{N}\right)_{N}^{k}
$$


Due to the inf-sup condition (22), for any data $\mathbf{f}$ continuous on $\bar{\Omega}$, this problem admits a unique solution.

Note, to conclude, that the integrals that appear in the definition $(16)$ of $b(\cdot, \cdot)$ can be replaced by the discrete product (18) for both choices of the pressure discrete spaces in the case of $\Omega_{k}$ being a straight edged quadrilateral. This, of course, is important for the implementation.

\section{An a posteriori error indicator}

Drawing our inspiration from the results of Verfürth $(1989,1991,1994)$; Bernardi (1996) and Bernardi et al. (0000), we define an elemental error indicator $\eta_{k}$ for the Stokes problem (1)-(3) thus:

$$
\begin{aligned}
\eta_{k}= & \frac{h_{k}}{N_{k}}\left\|\mathcal{I}_{N}^{k} \mathbf{f}+v \nabla^{2} \mathbf{u}_{N}-\nabla p_{N}\right\|_{L^{2}\left(\Omega_{k}\right)^{2}}+\left\|\boldsymbol{\nabla} \cdot \mathbf{u}_{N}\right\|_{L^{2}\left(\Omega_{k}\right)} \\
& +\frac{1}{2}\left(\frac{h_{k}}{N_{k}}\right)^{\frac{1}{2}} \sum_{l=1}^{L(k)}\left\|\left[v \frac{\partial \mathbf{u}_{N}}{\partial n}-p_{N} \mathbf{n}\right]\right\|_{L^{2}\left(\Gamma_{k, l}\right)^{2}}
\end{aligned}
$$

where $h_{k}$ denotes the diameter of $\Omega_{k}$ and $\mathcal{I}_{N}^{k}$ the Lagrange interpolation operator on the grid made of the $\left(x_{i}^{k}, y_{j}^{k}\right), 0 \leqslant i, j \leqslant N_{k}$. We use the notation [ ] in the last term on the righthand side of (24) to indicate a jump across the interface between two subdomains.

From now on, for simplicity, we assume that the aspect ratio of the $\Omega_{k}$, i.e. the ratio of $h_{k}$ to the length of the smallest edge of $\Omega_{k}, 1 \leqslant k \leqslant K$, is bounded by a constant and we do not take this constant into account. Let $\Delta_{k}, 1 \leqslant k \leqslant K$, denote the interior of the convex hull of the union of the spectral elements $\bar{\Omega}_{k^{\prime}}$ whose intersection with $\bar{\Omega}_{k}$ is non-empty. Let $N_{k+}$ denote the maximum of the $N_{k^{\prime}}$ for all $k^{\prime}$ such that $\Omega_{k^{\prime}}$ is contained in $\Delta_{k}$ and denote by $\lambda_{k}$ the largest ratio $N_{k^{\prime}} / N_{k^{\prime \prime}}$ for all pair of domains $\Omega_{k^{\prime}}$ and $\Omega_{k^{\prime \prime}}$ contained in $\Delta_{k}$. In an analogous fashion, let $h_{k+}$ denote the maximum of $h_{k^{\prime}}$ for all $k^{\prime}$ such that $\Omega_{k^{\prime}}$ is contained in $\Delta_{k}$ and denote by $\mu_{k}$ the quotient of $h_{k+}$ by $h_{k}$. Finally, let $v_{k}$ denote the number of subdomains $\Delta_{k^{\prime}}$ containing the same $\Omega_{k}$ and $\omega_{k}$ the union of the elements $\Omega_{k^{\prime}}$ such that $\partial \Omega_{k^{\prime}} \cap \partial \Omega_{k}$ has non-zero measure. We are now in a position to state our two main results.

THEOREM 1 Assume that the data $\mathbf{f}$ are such that each $\left.\mathbf{f}\right|_{\omega_{k}}, 1 \leqslant k \leqslant K$, belong to $H^{\sigma_{k}}\left(\omega_{k}\right)^{2}, \sigma_{k}>1$. Let (u, $\left.p\right)$ denote the exact solution to the Stokes problem (1)-(3), and $\left(\mathbf{u}_{N}, p_{N}\right)$ the non-conforming discrete solution of problem (23). If the domain $\Omega$ is convex and if the two following assumptions hold for $1 \leqslant k \leqslant K$ and $1 \leqslant l \leqslant L(k)$ :

$$
\begin{aligned}
& \lim _{N_{k} \rightarrow \infty} \frac{\inf _{\mathbf{v}_{N} \in \mathbb{P}_{N_{k}-1}\left(\Omega_{k}\right)}\left|\mathbf{u}_{N}-\mathbf{v}_{N}\right|_{H^{1}\left(\Omega_{k}\right)^{2}}}{\left|\mathbf{u}-\mathbf{u}_{N}\right|_{H^{1}\left(\Omega_{k}\right)^{2}}}=0, \\
& \inf _{\psi_{N} \in \mathbb{P}_{N_{k}-2}\left(\Gamma_{k, l}\right)}\left\|\nu \frac{\partial \mathbf{u}_{N}}{\partial n}-p_{N} \mathbf{n}-\psi_{N}\right\|_{L^{2}\left(\Gamma_{k, l}\right)^{2}} \leqslant c\left\|\left[v \frac{\partial \mathbf{u}_{N}}{\partial n}-p_{N} \mathbf{n}\right]\right\|_{L^{2}\left(\Gamma_{k, l}\right)^{2}},
\end{aligned}
$$

when all the $N_{k}$ are large enough, then there exists a constant $c_{1}$ independent of all the $N_{k}$, 
such that

$$
\begin{aligned}
\left|\mathbf{u}-\mathbf{u}_{N}\right|_{H^{1}\left(\cup \Omega_{k}\right)^{2}} & +\left\|p-p_{N}\right\|_{L^{2}(\Omega)} \\
& \leqslant c_{1}\left(\sum_{k=1}^{K}\left(\left(\lambda_{k} \mu_{k} v_{k} \eta_{k}\right)^{2}+\left(v_{k} h_{k}^{\min \left\{\sigma_{k}, N_{k}+1\right\}} N_{k}^{-\sigma_{k}}\|\mathbf{f}\|_{H^{\sigma_{k}}\left(\omega_{k}\right)^{2}}\right)^{2}\right)\right)^{\frac{1}{2}},
\end{aligned}
$$

for the error indicator $\eta^{k}$ defined in (24).

THEOREM 2 There exists a constant $c_{2}$ independent of all the $N_{k}$, such that each error indicator $\eta_{k}, 1 \leqslant k \leqslant K$, defined in (24) satisfies for any $\varepsilon>0$

$$
\begin{aligned}
\eta_{k} \leqslant & c_{2} \lambda_{k}^{3 / 2-\varepsilon} \mu_{k}^{1+\varepsilon}\left(N_{k}^{3 / 2+\varepsilon}\left(\left|\mathbf{u}-\mathbf{u}_{N}\right|_{H^{1}\left(\left(\cup \Omega_{k}\right) \cap \omega_{k}\right)^{2}}+\left\|p-p_{N}\right\|_{L^{2}\left(\omega_{k}\right)}\right)\right. \\
& \left.+h_{k} N_{k}^{2 \varepsilon}\left\|\mathbf{f}-\mathcal{I}_{N} \mathbf{f}\right\|_{L^{2}\left(\omega_{k}\right)^{2}}\right)
\end{aligned}
$$

where $\mathcal{I}_{N}$ now stands for the global interpolation operator, defined by $\left.\left(\mathcal{I}_{N} \mathbf{f}\right)\right|_{\Omega_{k}}=\mathcal{I}_{N}^{k} \mathbf{f} \mid \Omega_{k}$.

We now prove these theorems. We begin with estimate (27), which relies on the following argument. Let us first introduce the space $\tilde{U}_{N}\left(\cup \Omega_{k}\right)$ of functions $u$ such that

1. each $\left.u\right|_{\Omega_{k}}, 1 \leqslant k \leqslant K$, belongs to $H^{1}\left(\Omega_{k}\right)$,

2. $u$ vanishes on $\partial \Omega$,

3. the following matching condition holds on any edge $\Gamma_{k, l}, 1 \leqslant k \leqslant K, 1 \leqslant l \leqslant L(k)$,

$$
\forall \psi \in \mathbb{P}_{N_{k}-2}\left(\Gamma_{k, l}\right), \int_{\Gamma_{k, l}}\left(\left.u\right|_{\Omega_{k}}-\phi\right)(\tau) \psi(\tau) \mathrm{d} \tau=0,
$$

where the mortar function $\phi$ is defined on the skeleton $\mathcal{S}$ by

$$
\left.\phi\right|_{\gamma^{m}}=\left.\left.u\right|_{\Omega_{k(m)}}\right|_{\gamma^{m}}, \quad 1 \leqslant m \leqslant M .
$$

The idea is that the space $\tilde{X}_{N}(\Omega)=\tilde{U}_{N}(\Omega) \times \tilde{U}_{N}(\Omega)$ contains both spaces $X(\Omega)$ and $X_{N}$.

Indeed, it is proven in Ben Belgacem et al. (2000, Theorem A.2) that the form $a(\cdot, \cdot)$, extended to discontinuous functions by

$$
a(\mathbf{u}, \mathbf{v})=v \sum_{k=1}^{K} \int_{\Omega_{k}}(\nabla \mathbf{u})^{T}:(\nabla \mathbf{v}) \mathrm{d} x \mathrm{~d} y,
$$

still satisfies the ellipticity property

$$
\forall u \in \tilde{X}_{N}(\Omega), \quad a(u, u) \geqslant \tilde{\alpha}\|u\|_{H^{1}\left(\cup \Omega_{k}\right)^{2}},
$$

for a constant $\tilde{\alpha}$ only depending on the decomposition of $\Omega$ (more precisely on the maximal aspect ratio of the $\Omega_{k}$ ) but not on the $N_{k}$. Since the inf-sup condition (7) obviously holds with $X(\Omega)$ replaced by $\tilde{X}_{N}(\Omega)$, setting $\tilde{Z}_{N}(\Omega)=\tilde{X}_{N}(\Omega) \times L_{0}^{2}(\Omega)$, we derive the following extension of (8), for a positive constant $\tilde{\beta}_{2}$ :

$$
\forall(\mathbf{u}, p) \in \tilde{Z}_{N}(\Omega), \quad \operatorname{Sup}_{(\mathbf{v}, q) \in \tilde{Z}_{N}(\Omega)} \frac{\mathcal{L}((\mathbf{u}, p) ;(\mathbf{v}, q))}{\|(\mathbf{v}, q)\|_{Z}} \geqslant \tilde{\beta}_{2}\|(\mathbf{u}, p)\|_{Z} .
$$


Now we can apply this inequality to the pair $\left(\mathbf{u}-\mathbf{u}_{N}, p-p_{N}\right)$, which belongs to $\tilde{Z}_{N}(\Omega)$ :

$$
\left|\mathbf{u}-\mathbf{u}_{N}\right|_{H^{1}\left(\cup \Omega_{k}\right)^{2}}+\left\|p-p_{N}\right\|_{L^{2}(\Omega)} \leqslant \frac{1}{\tilde{\beta}_{2}} \operatorname{Sup}_{(\mathbf{v}, q) \in \tilde{Z}_{N}(\Omega)} \frac{\mathcal{L}\left(\left(\mathbf{u}-\mathbf{u}_{N}, p-p_{N}\right) ;(\mathbf{v}, q)\right)}{\|(\mathbf{v}, q)\|_{Z}} .
$$

Next, we observe that, for any $(\mathbf{v}, q)$ in $\tilde{Z}_{N}(\Omega)$,

$$
\mathcal{L}\left(\left(\mathbf{u}-\mathbf{u}_{N}, p-p_{N}\right) ;(\mathbf{v}, q)\right)=a\left(\mathbf{u}-\mathbf{u}_{N}, \mathbf{v}\right)+b\left(\mathbf{v}, p-p_{N}\right)+b\left(\mathbf{u}-\mathbf{u}_{N}, q\right),
$$

and we must evaluate all the terms on the right-hand side. The last one is obvious:

$$
b\left(\mathbf{u}-\mathbf{u}_{N}, q\right)=-b\left(\mathbf{u}_{N}, q\right) \leqslant\left\|\nabla \cdot \mathbf{u}_{N}\right\|_{L^{2}(\Omega)}\|q\|_{L^{2}(\Omega)} .
$$

We now proceed to multiply equation (1) by any function $\mathbf{v}_{N}$ in $X_{N}$. Integrating by parts, we observe that

$$
a\left(\mathbf{u}, \mathbf{v}_{N}\right)+b\left(\mathbf{v}_{N}, p\right)=\int_{\Omega} \mathbf{f} \cdot \mathbf{v}_{N} \mathrm{~d} x \mathrm{~d} y+\int_{\mathcal{S}}\left(v \frac{\partial \mathbf{u}}{\partial n}-p \mathbf{n}\right) \cdot\left[\mathbf{v}_{N}\right] \mathrm{d} \tau,
$$

so that, when combined with (23), this yields

$$
\begin{aligned}
a\left(\mathbf{u}-\mathbf{u}_{N}, \mathbf{v}\right)+b\left(\mathbf{v}, p-p_{N}\right)= & \sum_{k=1}^{K}\left(v \int_{\Omega_{k}}\left(\nabla\left(\mathbf{u}-\mathbf{u}_{N}\right)\right)^{T}: \nabla\left(\mathbf{v}-\mathbf{v}_{N}\right) \mathrm{d} x \mathrm{~d} y\right. \\
& -\int_{\Omega_{k}}\left(p-p_{N}\right)\left(\nabla \cdot\left(\mathbf{v}-\mathbf{v}_{N}\right)\right) \mathrm{d} x \mathrm{~d} y \\
& \left.+\int_{\Omega_{k}} \mathbf{f} \cdot \mathbf{v}_{N} \mathrm{~d} x \mathrm{~d} y-\left(\mathbf{f}, \mathbf{v}_{N}\right)_{N}^{k}\right) \\
& +a_{N}\left(\mathbf{u}_{N}, \mathbf{v}_{N}\right)-a\left(\mathbf{u}_{N}, \mathbf{v}_{N}\right)+\int_{\mathcal{S}}\left(v \frac{\partial \mathbf{u}}{\partial n}-p \mathbf{n}\right) \cdot\left[\mathbf{v}_{N}\right] \mathrm{d} \tau .
\end{aligned}
$$

Integrating by parts leads to

$$
\begin{aligned}
a\left(\mathbf{u}-\mathbf{u}_{N}, \mathbf{v}\right)+b\left(\mathbf{v}, p-p_{N}\right)= & \sum_{k=1}^{K}\left(\int_{\Omega_{k}}\left(\mathcal{I}_{N}^{k} \mathbf{f}+v \nabla^{2} \mathbf{u}_{N}-\nabla p_{N}\right) \cdot\left(\mathbf{v}-\mathbf{v}_{N}\right) \mathrm{d} x \mathrm{~d} y\right. \\
& +\int_{\Omega_{k}}\left(\mathbf{f}-\mathcal{I}_{N}^{k} \mathbf{f}\right) \cdot\left(\mathbf{v}-\mathbf{v}_{N}\right) \mathrm{d} x \mathrm{~d} y \\
& \left.+\int_{\Omega_{k}} \mathbf{f} \cdot \mathbf{v}_{N} \mathrm{~d} x \mathrm{~d} y-\left(\mathbf{f}, \mathbf{v}_{N}\right)_{N}^{k}\right) \\
& +a_{N}\left(\mathbf{u}_{N}, \mathbf{v}_{N}\right)-a\left(\mathbf{u}_{N}, \mathbf{v}_{N}\right)+\int_{\mathcal{S}}\left(v \frac{\partial \mathbf{u}}{\partial n}-p \mathbf{n}\right) \cdot\left[\mathbf{v}_{N}\right] \mathrm{d} \tau \\
& -\int_{\mathcal{S}}\left[v \frac{\partial \mathbf{u}_{N}}{\partial n}-p_{N} \mathbf{n}\right] \cdot\left(\mathbf{v}-\mathbf{v}_{N}\right) \mathrm{d} \tau \\
& +\int_{\mathcal{S}}\left(v \frac{\partial\left(\mathbf{u}-\mathbf{u}_{N}\right)}{\partial n}-\left(p-p_{N}\right) \mathbf{n}\right) \cdot\left[\mathbf{v}-\mathbf{v}_{N}\right] \mathrm{d} \tau
\end{aligned}
$$


or, equivalently,

$$
\begin{aligned}
a\left(\mathbf{u}-\mathbf{u}_{N}, \mathbf{v}\right)+b\left(\mathbf{v}, p-p_{N}\right)= & \sum_{k=1}^{K}\left(\int_{\Omega_{k}}\left(\mathcal{I}_{N}^{k} \mathbf{f}+v \nabla^{2} \mathbf{u}_{N}-\nabla p_{N}\right) \cdot\left(\mathbf{v}-\mathbf{v}_{N}\right) \mathrm{d} x \mathrm{~d} y\right. \\
& +\int_{\Omega_{k}}\left(\mathbf{f}-\mathcal{I}_{N}^{k} \mathbf{f}\right) \cdot\left(\mathbf{v}-\mathbf{v}_{N}\right) \mathrm{d} x \mathrm{~d} y \\
& \left.+\int_{\Omega_{k}} \mathbf{f} \cdot \mathbf{v}_{N} \mathrm{~d} x \mathrm{~d} y-\left(\mathbf{f}, \mathbf{v}_{N}\right)_{N}^{k}\right) \\
& +a_{N}\left(\mathbf{u}_{N}, \mathbf{v}_{N}\right)-a\left(\mathbf{u}_{N}, \mathbf{v}_{N}\right)+\int_{\mathcal{S}}\left(v \frac{\partial \mathbf{u}}{\partial n}-p \mathbf{n}\right) \cdot[\mathbf{v}] \mathrm{d} \tau \\
& -\int_{\mathcal{S}}\left[v \frac{\partial \mathbf{u}_{N}}{\partial n}-p_{N} \mathbf{n}\right] \cdot\left(\mathbf{v}-\mathbf{v}_{N}\right) \mathrm{d} \tau \\
& -\int_{\mathcal{S}}\left(v \frac{\partial \mathbf{u}_{N}}{\partial n}-p_{N} \mathbf{n}\right) \cdot\left[\mathbf{v}-\mathbf{v}_{N}\right] \mathrm{d} \tau
\end{aligned}
$$

It remains to evaluate all the terms on the right-hand side of (35).

We first recall the standard estimate (Bernardi \& Maday, 1996, Theorem 14.2): if the function $\mathbf{f}$ is such that $\left.\mathbf{f}\right|_{\Omega_{k}}$ belong to $H^{\sigma_{k}}\left(\Omega_{k}\right)^{2}, \sigma_{k}>1$,

$$
\left\|\mathbf{f}-\mathcal{I}_{N}^{k} \mathbf{f}\right\|_{L^{2}\left(\Omega_{k}\right)^{2}} \leqslant c h_{k}^{\min \left\{\sigma_{k}, N_{k}+1\right\}} N_{k}^{-\sigma_{k}}\|\mathbf{f}\|_{H^{\sigma_{k}}\left(\Omega_{k}\right)^{2}} .
$$

This yields in an obvious way

$$
\left|\int_{\Omega_{k}}\left(\mathbf{f}-\mathcal{I}_{N}^{k} \mathbf{f}\right) \cdot\left(\mathbf{v}-\mathbf{v}_{N}\right) \mathrm{d} x \mathrm{~d} y\right| \leqslant c h_{k}^{\min \left\{\sigma_{k}, N_{k}+1\right\}} N_{k}^{-\sigma_{k}}\|\mathbf{f}\|_{H^{\sigma_{k}}\left(\Omega_{k}\right)^{2}}\left\|\mathbf{v}-\mathbf{v}_{N}\right\|_{H^{1}\left(\Omega_{k}\right)^{2}} .
$$

Similarly, by adding and subtracting the image of $\mathbf{f}$ by the orthogonal projection operator from $L^{2}\left(\Omega_{k}\right)$ onto $\mathbb{P}_{N_{k}-1}\left(\Omega_{k}\right)$, we derive

$$
\left|\int_{\Omega_{k}} \mathbf{f} \cdot \mathbf{v}_{N} \mathrm{~d} x \mathrm{~d} y-\left(\mathbf{f}, \mathbf{v}_{N}\right)_{N}^{k}\right| \leqslant c h_{k}^{\min \left\{\sigma_{k}, N_{k}+1\right\}} N_{k}^{-\sigma_{k}}\|\mathbf{f}\|_{H^{\sigma_{k}}\left(\Omega_{k}\right)^{2}}\left\|\mathbf{v}_{N}\right\|_{H^{1}\left(\Omega_{k}\right)^{2}} .
$$

The fifth term is estimated in the next lemma.

Lemma 1 Assume that the data $\mathbf{f}$ is such that each $\left.\mathbf{f}\right|_{\omega_{k}}, 1 \leqslant k \leqslant K$, belongs to $H^{\sigma_{k}}\left(\omega_{k}\right)^{2}$, $\sigma_{k} \geqslant 0$. If $\Omega$ is convex, there exists a constant $c$ independent of the $h_{k}$ and $N_{k}$ such that

$$
\begin{aligned}
\mid \int_{\mathcal{S}}\left(v \frac{\partial \mathbf{u}}{\partial n}-p \mathbf{n}\right) & \cdot[\mathbf{v}] \mathrm{d} \tau \mid \\
& \leqslant c\left(\sum_{k=1}^{K}\left(h_{k}^{\min \left\{\sigma_{k}+1, N_{k}+1\right\}} N_{k}^{-\sigma_{k}-1}\|\mathbf{f}\|_{H^{\sigma_{k}}\left(\omega_{k}\right)^{2}}\right)^{2}\right)^{\frac{1}{2}}\|\mathbf{v}\|_{H^{1}\left(\cup \Omega_{k}\right)^{2}}
\end{aligned}
$$


Proof. On each $\Gamma_{k, l}$ which is not a mortar, we have by definition of the space $\tilde{X}_{N}(\Omega)$

$$
\begin{aligned}
\int_{\Gamma_{k, l}}\left(v \frac{\partial \mathbf{u}}{\partial n}-p \mathbf{n}\right) \cdot[\mathbf{v}] \mathrm{d} \tau & =\int_{\Gamma_{k, l}}\left(\mathrm{i} d-\pi_{N-2}^{k, l}\right)\left(v \frac{\partial \mathbf{u}}{\partial n}-p \mathbf{n}\right) \cdot[\mathbf{v}] \mathrm{d} \tau \\
& \leqslant\left\|\left(\mathrm{i} d-\pi_{N-2}^{k, l}\right)\left(v \frac{\partial \mathbf{u}}{\partial n}-p \mathbf{n}\right)\right\|_{H^{-\frac{1}{2}}\left(\Gamma_{k, l}\right)^{2}}\|[\mathbf{v}]\|_{H^{\frac{1}{2}}\left(\Gamma_{k, l}\right)^{2}},
\end{aligned}
$$

where $\pi_{N-2}^{k, l}$ stands for the orthogonal projection operator from $L^{2}\left(\Gamma_{k, l}\right)$ onto $\mathbb{P}_{N_{k}-2}\left(\Gamma_{k, l}\right)$. With obvious definition of the norm $\|\cdot\|_{H^{1}\left(\cup \Omega_{k} ; \omega_{k}\right)}$ by restriction to $\omega_{k}$, it is readily checked that

$$
\|[\mathbf{v}]\|_{H^{\frac{1}{2}}\left(\Gamma_{k, l}\right)^{2}} \leqslant\|\mathbf{v}\|_{H^{1}\left(\cup \Omega_{k} ; \omega_{k}\right)^{2}} .
$$

Next, we recall the standard property of the operator $\pi_{N-2}^{k, l}$ : for any $\phi$ in $H^{t}\left(\Gamma_{k, l}\right), t \geqslant 0$,

$$
\left\|\phi-\pi_{N-2}^{k, l} \phi\right\|_{H^{-\frac{1}{2}}\left(\Gamma_{k, l}\right)} \leqslant c h_{k}^{\min \left\{t+\frac{1}{2}, N_{k}+1\right\}} N_{k}^{-t-\frac{1}{2}}\|\phi\|_{H^{t}\left(\Gamma_{k, l}\right)} .
$$

We conclude by noting that, since $\Omega$ is convex, $\bar{\Gamma}_{k, l}$ does not contain any corner of $\Omega$, so that the solution $(\mathbf{u}, p)$ in a neighbourhood $\mathcal{O}$ of $\Gamma_{k, l}$ belongs to $H^{\sigma_{k}+2}(\mathcal{O})^{2} \times H^{\sigma_{k}+1}(\mathcal{O})$. As a consequence, $v \frac{\partial \mathbf{u}}{\partial n}-p \mathbf{n}$ belongs to $H^{\sigma_{k}+\frac{1}{2}}\left(\Gamma_{k, l}\right)^{2}$ and using the result

$$
\left\|\nu \frac{\partial \mathbf{u}}{\partial n}-p \mathbf{n}\right\|_{H^{\sigma_{k}+\frac{1}{2}}\left(\Gamma_{k, l}\right)^{2}} \leqslant c\|\mathbf{f}\|_{H^{\sigma_{k}\left(\omega_{k}\right)^{2}}},
$$

the desired estimate follows.

Thanks to the exactness of the quadrature formula, we have for all $\mathbf{w}_{N}^{k}$ in $\mathbb{P}_{N_{k}-1}\left(\Omega_{k}\right)$,

$$
\left|a_{N}\left(\mathbf{u}_{N}, \mathbf{v}_{N}\right)-a\left(\mathbf{u}_{N}, \mathbf{v}_{N}\right)\right| \leqslant \sum_{k=1}^{K}\left\|\mathbf{u}_{N}-\mathbf{w}_{N}^{k}\right\|_{H^{1}\left(\Omega_{k}\right)}\left\|\mathbf{v}_{N}\right\|_{H^{1}\left(\Omega_{k}\right)} .
$$

So, appealing to Hypothesis (25) and using it to bound the right-hand side of (42) we may choose each $N_{k}$ large enough such that

$$
\left|a_{N}\left(\mathbf{u}_{N}, \mathbf{v}_{N}\right)-a\left(\mathbf{u}_{N}, \mathbf{v}_{N}\right)\right| \leqslant c \tilde{\beta}_{2}\left|\mathbf{u}-\mathbf{u}_{N}\right|_{H^{1}\left(\cup \Omega_{k}\right)^{2}}\left\|\mathbf{v}_{N}\right\|_{H^{1}\left(\cup \Omega_{k}\right)^{2}},
$$

for some $c<1$. We also employ Hypothesis (26) to bound the last term in (35). Inserting all 
this in (35) and combining the result with (32) and (34) yields, after some rearrangement,

$$
\begin{aligned}
\left|\mathbf{u}-\mathbf{u}_{N}\right|_{H^{1}\left(\cup \Omega_{k}\right)^{2}} & +\left\|p-p_{N}\right\|_{L^{2}(\Omega)} \\
\leqslant & c\left(\sum _ { k = 1 } ^ { K } \left(\left\|\mathcal{I}_{N}^{k} \mathbf{f}+v \nabla^{2} \mathbf{u}_{N}-\nabla p_{N}\right\|_{L^{2}\left(\Omega_{k}\right)^{2}} \frac{\left\|\mathbf{v}-\mathbf{v}_{N}\right\|_{L^{2}\left(\Omega_{k}\right)^{2}}}{|\mathbf{v}|_{H^{1}\left(\cup \Omega_{k}\right)^{2}}}\right.\right. \\
& \left.+\frac{1}{2} \sum_{l=1}^{L(k)}\left\|\left[v \frac{\partial \mathbf{u}_{N}}{\partial n}-p_{N} \mathbf{n}\right]\right\|_{L^{2}\left(\Gamma_{k, l}\right)^{2}} \frac{\left\|\mathbf{v}-\mathbf{v}_{N}\right\|_{L^{2}\left(\Gamma_{k, l}\right)^{2}}}{|\mathbf{v}|_{H^{1}\left(\cup \Omega_{k}\right)^{2}}}\right) \\
& +\left\|\boldsymbol{\nabla} \cdot \mathbf{u}_{N}\right\|_{L^{2}(\Omega)} \\
& +\left(\sum _ { k = 1 } ^ { K } ( h _ { k } ^ { \operatorname { m i n } \{ \sigma _ { k } , N _ { k } + 1 \} } N _ { k } ^ { - \sigma _ { k } } \| \mathbf { f } \| _ { H ^ { \sigma _ { k } } ( \Omega _ { k } ) ^ { 2 } ) ^ { 2 } } ) ^ { \frac { 1 } { 2 } } \left(1+\frac{\left.\left.\left|\mathbf{v}_{N}\right|_{H^{1}\left(\cup \Omega_{k}\right)^{2}}\right)\right),}{\left.|\mathbf{v}|_{H^{1}\left(\cup \Omega_{k}\right)^{2}}\right)}\right.\right.
\end{aligned}
$$

for some constant c. So, it remains to exhibit an appropriate approximation $\mathbf{v}_{N}$ of $\mathbf{v}$ in $X_{N}$. We need a preliminary lemma concerning the orthogonal projection operator $\Pi_{N}^{1,0}$ from $\tilde{X}_{N}(\Omega)$ onto $X_{N}$ for the scalar product associated with the norm $|\cdot|_{H^{1}\left(\cup \Omega_{k}\right)^{2}}$.

LEMMA 2 When $\Omega$ is convex, the following estimates hold for any function $\mathbf{v}$ in $\tilde{X}_{N}(\Omega)$ :

$$
\left|\Pi_{N}^{1,0} \mathbf{v}\right|_{H^{1}\left(\cup \Omega_{k}\right)^{2}} \leqslant|\mathbf{v}|_{H^{1}\left(\cup \Omega_{k}\right)^{2}},
$$

and

$$
\begin{gathered}
\left\|\mathbf{v}-\Pi_{N}^{1,0} \mathbf{v}\right\|_{L^{2}\left(\Omega_{k}\right)^{2}} \leqslant c \max _{1 \leqslant k \leqslant K}\left\{h_{k} N_{k}^{-1}\right\}|\mathbf{v}|_{H^{1}\left(\cup \Omega_{k}\right)^{2}}, \\
\left\|\mathbf{v}-\Pi_{N}^{1,0} \mathbf{v}\right\|_{L^{2}(\mathcal{S})^{2}} \leqslant c \max _{1 \leqslant k \leqslant K}\left\{h_{k}^{\frac{1}{2}} N_{k}^{-\frac{1}{2}}\right\}|\mathbf{v}|_{H^{1}\left(\cup \Omega_{k}\right)^{2} .}
\end{gathered}
$$

Proof. Estimate (44) is obvious thanks to the definition of the operator $\Pi_{N}^{1,0}$. In order to prove (45), we use the duality equation

$$
\left\|\mathbf{v}-\Pi_{N}^{1,0} \mathbf{v}\right\|_{L^{2}(\Omega)^{2}}=\sup _{\mathbf{g} \in L^{2}(\Omega)^{2}} \frac{\int_{\Omega}\left(\mathbf{v}-\Pi_{N}^{1,0} \mathbf{v}\right) \cdot \mathbf{g d} x \mathrm{~d} y}{\|\mathbf{g}\|_{L^{2}(\Omega)^{2}}} .
$$

Indeed, for any $\mathbf{g}$ in $L^{2}(\Omega)^{2}$, we consider the problem

$$
\begin{aligned}
-\nabla^{2} \mathbf{w}=\mathbf{g}, & \text { in } \Omega, \\
\mathbf{w}=\mathbf{0}, & \text { on } \partial \Omega,
\end{aligned}
$$

and we observe that, since $\Omega$ is convex, it has a unique solution w in $H_{0}^{1}(\Omega)^{2} \cap H^{2}(\Omega)^{2}$ which satisfies

$$
\|\mathbf{w}\|_{H^{2}(\Omega)^{2}} \leqslant c\|\mathbf{g}\|_{L^{2}(\Omega)^{2}}
$$


From the definition of $\tilde{X}_{N}(\Omega)$ and using (41) we see that

$$
\begin{aligned}
\int_{\Gamma_{k, l}}\left[\mathbf{v}-\Pi_{N}^{1,0} \mathbf{v}\right] \cdot \frac{\partial \mathbf{w}}{\partial n} \mathrm{~d} \tau & =\int_{\Gamma_{k, l}}\left[\mathbf{v}-\Pi_{N}^{1,0} \mathbf{v}\right] \cdot\left(\mathrm{i} d-\pi_{N-2}^{k, l}\right) \frac{\partial \mathbf{w}}{\partial n} \mathrm{~d} \tau, \\
& \leqslant\left\|\left[\mathbf{v}-\Pi_{N}^{1,0} \mathbf{v}\right]\right\|_{H^{\frac{1}{2}}\left(\Gamma_{k, l}\right)^{2}} h_{k} N_{k}^{-1}\left\|\frac{\partial \mathbf{w}}{\partial n}\right\|_{H^{\frac{1}{2}}\left(\Gamma_{k, l}\right)^{2}} .
\end{aligned}
$$

So, we have by integrating by parts and using the definition of $\Pi_{N}^{1,0}$

$$
\begin{aligned}
\int_{\Omega}\left(\mathbf{v}-\Pi_{N}^{1,0} \mathbf{v}\right) \cdot \mathbf{g} \mathrm{d} x \mathrm{~d} y= & \sum_{k=1}^{K} \int_{\Omega_{k}} \nabla\left(\mathbf{v}-\Pi_{N}^{1,0} \mathbf{v}\right) \cdot \nabla \mathbf{w} \mathrm{d} x \mathrm{~d} y-\int_{\mathcal{S}}\left[\mathbf{v}-\Pi_{N}^{1,0} \mathbf{v}\right] \cdot \frac{\partial \mathbf{w}}{\partial n} \mathrm{~d} \tau \\
= & \sum_{k=1}^{K} \int_{\Omega_{k}} \nabla \mathbf{v} \cdot \nabla\left(\mathbf{w}-\Pi_{N}^{1,0} \mathbf{w}\right) \mathrm{d} x \mathrm{~d} y-\int_{\mathcal{S}}\left[\mathbf{v}-\Pi_{N}^{1,0} \mathbf{v}\right] \cdot \frac{\partial \mathbf{w}}{\partial n} \mathrm{~d} \tau \\
\leqslant & |\mathbf{v}|_{H^{1}\left(\cup \Omega_{k}\right)^{2}}\left|\mathbf{w}-\Pi_{N}^{1,0} \mathbf{w}\right|_{H^{1}\left(\cup \Omega_{k}\right)^{2}} \\
& +\left\|\mathbf{v}-\Pi_{N}^{1,0} \mathbf{v}\right\|_{H^{1}\left(\cup \Omega_{k}\right)^{2}} \max _{1 \leqslant k \leqslant K}\left\{h_{k} N_{k}^{-1}\right\}\|\mathbf{g}\|_{L^{2}(\Omega)^{2}} \\
\leqslant & c|\mathbf{v}|_{H^{1}\left(\cup \Omega_{k}\right)^{2}}\left(\left|\mathbf{w}-\Pi_{N}^{1,0} \mathbf{w}\right|_{H^{1}\left(\cup \Omega_{k}\right)^{2}}\right. \\
& \left.+\max _{1 \leqslant k \leqslant K}\left\{h_{k} N_{k}^{-1}\right\}\|\mathbf{g}\|_{L^{2}(\Omega)^{2}}\right)
\end{aligned}
$$

We derive estimate (45) by then combining the previous lines with the standard estimate (Bernardi et al., 1994)

$$
\left|\mathbf{w}-\Pi_{N}^{1,0} \mathbf{w}\right|_{H^{1}\left(\cup \Omega_{k}\right)^{2}} \leqslant c \max _{1 \leqslant k \leqslant K}\left\{h_{k} N_{k}^{-1}\right\}\|\mathbf{w}\|_{H^{2}(\Omega)^{2}} .
$$

Finally, we refer to Bernardi (1996, Corollary 2.6) for the proof of (46), which relies on a slightly more technical duality argument.

The proof of the next lemma relies on an idea of Bernardi (1996).

Lemma 3 When $\Omega$ is convex, for any function $\mathbf{v}$ in $\tilde{X}_{N}(\Omega)$, there exists a $\mathbf{v}_{N}$ in $X_{N}$ such that the following estimates hold:

$$
\left\|\mathbf{v}_{N}\right\|_{H^{1}\left(\cup \Omega_{k}\right)^{2}} \leqslant v_{k}\|\mathbf{v}\|_{H^{1}\left(\cup \Omega_{k}\right)^{2}}
$$

and, for $1 \leqslant k \leqslant K$ and $1 \leqslant l \leqslant L(k)$,

$$
\begin{gathered}
\left\|\mathbf{v}-\mathbf{v}_{N}\right\|_{L^{2}(\Omega)^{2}} \leqslant c \lambda_{k} \mu_{k} h_{k} N_{k}^{-1}|\mathbf{v}|_{H^{1}\left(\cup \Omega_{k} ; \Delta_{k}\right)^{2}}, \\
\left\|\mathbf{v}-\Pi_{N}^{1,0} \mathbf{v}\right\|_{L^{2}\left(\Gamma_{k, l}\right)^{2}} \leqslant c \lambda_{k} \mu_{k} h_{k}^{\frac{1}{2}} N_{k}^{-\frac{1}{2}}|\mathbf{v}|_{H^{1}\left(\cup \Omega_{k} ; \Delta_{k}\right)^{2},}
\end{gathered}
$$

where $|\cdot|_{H^{1}\left(\cup \Omega_{k} ; \Delta_{k}\right)}$ denotes the restriction to $\Delta_{k}$ of the norm $|\cdot|_{H^{1}\left(\cup \Omega_{k}\right)}$.

Proof. We introduce a partition of unity on $\bar{\Omega}$ by continuously differentiable functions $\psi_{k}$ with values in $[0,1]$, which have support in $\Delta_{k}$ and are $\geqslant \frac{1}{2}$ over $\Omega_{k}$. Let $X_{N}^{k}$ and $\tilde{X}_{N}\left(\Delta_{k}\right)$ 
denote the space of restrictions to $\Delta_{k}$ of functions in $X_{N}$ and $\tilde{X}_{N}(\Omega)$, respectively, which vanish on $\partial \Omega_{k}$. The idea is to choose

$$
\mathbf{v}_{N}=\sum_{k=1}^{K} \Pi_{N, k}^{1,0}\left(\psi_{k} \mathbf{v}\right)
$$

where $\Pi_{N, k}^{1,0}$ denotes the orthogonal projection operator from $\tilde{X}_{N}\left(\Delta_{k}\right)$ onto $X_{N}^{k}$ for the scalar product associated with the norm $|\cdot|_{H^{1}\left(\cup \Omega_{k} ; \Delta_{k}\right)}$. Then, the desired estimates follow from Lemma 2 in an obvious way.

Inserting (49) to (51) into (43) leads to Theorem 1.

REMARK Assumptions (25) and (26) are saturation type hypotheses and seem to hold in a large number of practical situations. Moreover, assumption (25) can be avoided by using a higher order quadrature formula (namely, exact on $\mathbb{P}_{2 N_{k}}\left(\Omega_{k}\right)$ instead of $\mathbb{P}_{2 N_{k}-1}\left(\Omega_{k}\right)$, $1 \leqslant k \leqslant K$ ) or also if $\mathbb{P}_{N_{k}-2}(\Gamma)$ were replaced by $\mathbb{P}_{N_{k}-3}(\Gamma)$ in the matching condition that appears in the definition of $X_{N}(\Omega)$.

REMARK An analogous estimate to (27) holds when $\Omega$ is not convex. However it requires some further arguments in order to handle the singularities of the solution near the nonconvex corners, see Bernardi \& Maday (1991). It reads

$$
\begin{aligned}
& \left|\mathbf{u}-\mathbf{u}_{N}\right|_{H^{1}\left(\cup \Omega_{k}\right)^{2}}+\left\|p-p_{N}\right\|_{L^{2}(\Omega)} \\
\leqslant & c_{1}\left(\sum_{k=1}^{K}\left(\left(\lambda_{k} \mu_{k} v_{k} h_{k}^{-\beta_{k}} \eta_{k}\right)^{2}+\left(v_{k} h_{k}^{\min \left\{\sigma_{k}^{*}, N_{k}+1\right\}} N_{k}^{-\sigma_{k}^{*}}\|\mathbf{f}\|_{H^{\sigma_{k}}\left(\omega_{k}\right)^{2}}\right)^{2}\right)\right)^{\frac{1}{2}},
\end{aligned}
$$

where $\beta_{k}$ and $\sigma_{k}^{*}$ are equal to zero and $\sigma_{k}$, respectively, when $\bar{\Omega}_{k}$ does not contain any non-convex corner of $\Omega$, and to $\frac{1}{3}$ and $\eta\left(\frac{3 \pi}{2}\right)$, respectively, when $\bar{\Omega}_{k}$ contains a non-convex corner, where $\eta\left(\frac{3 \pi}{2}\right)$ is given in (9).

We are now interested in proving Theorem 2. Establishing inequality (28) relies on the equation, valid for any $(\mathbf{v}, q) \in Z(\Omega)$,

$$
\begin{aligned}
\sum_{k=1}^{K}\left(\int _ { \Omega _ { k } } \left(\mathcal{I}_{N}^{k} \mathbf{f}\right.\right. & \left.+v \nabla^{2} \mathbf{u}_{N}-\nabla p_{N}\right) \cdot \mathbf{v} \mathrm{d} x \mathrm{~d} y \\
& \left.-\frac{1}{2} \sum_{l=1}^{L(k)} \int_{\Gamma_{k, l}}\left[v \frac{\partial \mathbf{u}_{N}}{\partial n}-p_{N} \mathbf{n}\right] \cdot \mathbf{v} \mathrm{d} \tau+\int_{\Omega_{k}} q \boldsymbol{\nabla} \cdot \mathbf{u}_{N} \mathrm{~d} x \mathrm{~d} y\right) \\
& =\mathcal{L}\left(\left(\mathbf{u}-\mathbf{u}_{N}, p-p_{N}\right) ;(\mathbf{v}, q)\right)-\sum_{k=1}^{K} \int_{\Omega_{k}}\left(\mathbf{f}-\mathcal{I}_{N}^{k} \mathbf{f}\right) \cdot \mathbf{v} \mathrm{d} x \mathrm{~d} y .
\end{aligned}
$$

Our strategy in proving the desired result will be to bound successively each of the terms appearing on the right-hand side of (24) by choosing $\mathbf{v}$ appropriately. We first introduce some lemmas which will be required repeatedly in this section. 
Let us first recall the inequality, valid for any real numbers $\alpha$ and $\beta,-1<\beta \leqslant \alpha$, and for any sufficiently smooth function $\phi$,

$$
\int_{-1}^{1} \phi^{2}(\zeta)\left(1-\zeta^{2}\right)^{\alpha} \mathrm{d} \zeta \leqslant \int_{-1}^{1} \phi^{2}(\zeta)\left(1-\zeta^{2}\right)^{\beta} \mathrm{d} \zeta
$$

which is obvious since $1-\zeta^{2}$ is $\leqslant 1$ for $\zeta$ in $[0,1]$. The inverse inequality for polynomials is stated in the next lemma. Its proof requires the use of the Jacobi polynomials $J_{n}^{\alpha, \alpha}$ which are orthogonal to each other on $]-1,1\left[\right.$ for the measure $\left(1-\zeta^{2}\right)^{\alpha} \mathrm{d} \zeta$.

Lemma 4 Let $\alpha$ and $\beta$ be two real numbers such that $-1<\alpha<\beta$. Then the following inverse inequality holds for any polynomial $\phi_{N}$ in $\mathbb{P}_{N}[-1,1]$ :

$$
\int_{-1}^{1} \phi_{N}^{2}(\zeta)\left(1-\zeta^{2}\right)^{\alpha} \mathrm{d} \zeta \leqslant c N^{2(\beta-\alpha)} \int_{-1}^{1} \phi_{N}^{2}(\zeta)\left(1-\zeta^{2}\right)^{\beta} \mathrm{d} \zeta
$$

for a constant $c$ independent of $N$.

Proof. We recall (see for example Bernardi \& Maday, 1996, Section 19) that the derivatives $J_{n}^{\alpha, \alpha \prime}$ of the Jacobi polynomials are orthogonal to each other for the measure $\left(1-\zeta^{2}\right)^{\alpha+1} \mathrm{~d} \zeta$ and satisfy

$$
\int_{-1}^{1}\left(J_{n}^{\alpha, \alpha \prime}\right)^{2}(\zeta)\left(1-\zeta^{2}\right)^{\alpha+1} \mathrm{~d} \zeta=n(n+2 \alpha+1) \int_{-1}^{1}\left(J_{n}^{\alpha, \alpha}\right)^{2}(\zeta)\left(1-\zeta^{2}\right)^{\alpha} \mathrm{d} \zeta \geqslant c\left(n+\frac{1}{2}\right) .
$$

So, writing each $\phi_{N}$ in $\mathbb{P}_{N}[-1,1]$ as $\sum_{n=1}^{N+1} \alpha_{n} J_{n}^{\alpha, \alpha \prime}$, we have

$$
\int_{-1}^{1} \phi_{N}^{2}(\zeta)\left(1-\zeta^{2}\right)^{\alpha+1} \mathrm{~d} \zeta \geqslant c \sum_{n=1}^{N+1} \alpha_{n}^{2}\left(n+\frac{1}{2}\right)
$$

Next, it may be proved from Bernardi \& Maday (1996, Theorem 19.3) that

$$
\int_{-1}^{1}\left(J_{n}^{\alpha, \alpha \prime}\right)^{2}(\zeta)\left(1-\zeta^{2}\right)^{\alpha} \mathrm{d} \zeta \leqslant c n^{2}
$$

This yields

$$
\begin{aligned}
\int_{-1}^{1} \phi_{N}^{2}(\zeta)\left(1-\zeta^{2}\right)^{\alpha} \mathrm{d} \zeta & \leqslant c\left(\sum_{n=1}^{N+1}\left|\alpha_{n}\right|\left(\int_{-1}^{1}\left(J_{n}^{\alpha, \alpha \prime}\right)^{2}(\zeta)\left(1-\zeta^{2}\right)^{\alpha} \mathrm{d} \zeta\right)^{\frac{1}{2}}\right)^{2} \\
& \leqslant c\left(\sum_{n=1}^{N+1}\left|\alpha_{n}\right| n\right)^{2} .
\end{aligned}
$$

Using a Cauchy-Schwarz inequality now gives

$$
\begin{aligned}
\int_{-1}^{1} \phi_{N}^{2}(\zeta)\left(1-\zeta^{2}\right)^{\alpha} \mathrm{d} \zeta & \leqslant c\left(\sum_{n=1}^{N+1} \alpha_{n}^{2}\left(n+\frac{1}{2}\right)\right)\left(\sum_{n=1}^{N+1} \frac{n^{2}}{n+\frac{1}{2}}\right) \\
& \leqslant c^{\prime} N^{2} \int_{-1}^{1} \phi_{N}^{2}(\zeta)\left(1-\zeta^{2}\right)^{\alpha+1} \mathrm{~d} \zeta .
\end{aligned}
$$


So, we have proved (55) for $\beta=\alpha+1$. Iterating the argument, we therefore see that the result is true when the difference between $\beta$ and $\alpha$ is a positive integer. Finally, when $\beta-1<\alpha<\beta$, i.e. $\alpha$ can be written $\alpha=\frac{\beta-1}{p}+\frac{\beta}{p^{\prime}}$, with $\frac{1}{p}+\frac{1}{p^{\prime}}=1$ and $\frac{1}{p}=\beta-\alpha<1$, the result is proven by applying Hölder's inequality to

$$
\int_{-1}^{1} \phi_{N}^{2}(\zeta)\left(1-\zeta^{2}\right)^{\alpha} \mathrm{d} \zeta=\int_{-1}^{1} \phi_{N}^{\frac{2}{p}}(\zeta)\left(1-\zeta^{2}\right)^{\frac{\beta-1}{p}} \phi_{N}^{\frac{2}{p^{\prime}}}(\zeta)\left(1-\zeta^{2}\right)^{\frac{\beta}{p^{\prime}}} \mathrm{d} \zeta
$$

and using estimate (55) between $\beta-1$ and $\beta$. This completes the proof for any real $\beta>\alpha$.

The lemma which follows is dealt with by Bernardi (1996, formula (2.27)) and is simply stated here.

Lemma 5 Let $\alpha$ be a non-negative real number. Then the following inverse inequality holds for any polynomial $\phi_{N}$ in $\mathbb{P}_{N}(-1,1)$ :

$$
\int_{-1}^{1} \phi_{N}^{\prime 2}(\zeta)\left(1-\zeta^{2}\right)^{2 \alpha} \mathrm{d} \zeta \leqslant c N^{2(2-\alpha)} \int_{-1}^{1} \phi_{N}^{2}(\zeta)\left(1-\zeta^{2}\right)^{\alpha} \mathrm{d} \zeta
$$

for a constant $c$ independent of $N$.

For $1 \leqslant k \leqslant K$, we denote by $d_{k}$ the function defined in $\bar{\Omega}_{k}$ equal to the product of the distances to the four edges. For instance, if $\Omega_{k}$ stands for the rectangle $] a_{k}, b_{k}[\times] c_{k}, d_{k}[$, then the function $d_{k}$ is given by

$$
d_{k}(x, y)=\left(x-a_{k}\right)\left(b_{k}-x\right)\left(y-c_{k}\right)\left(d_{k}-y\right) .
$$

In order to apply the previous inequalities (55) and (56), we use a transformation that maps $\Omega_{k}$ onto the reference element $\left.\widehat{\Omega}=\right]-1,1\left[{ }^{2}\right.$, where the corresponding function $\hat{d}$ is defined by

$$
\hat{d}(\zeta, \xi)=\left(1-\zeta^{2}\right)\left(1-\xi^{2}\right)
$$

For a fixed $k$, we begin by choosing, for some $\beta, \frac{1}{2}<\beta \leqslant 2$,

$$
(\mathbf{v}, q)= \begin{cases}\left(\left(\mathcal{I}_{N}^{k} \mathbf{f}+v \nabla^{2} \mathbf{u}_{N}-\nabla p_{N}\right) d_{k}^{\beta}, 0\right) & \text { in } \Omega_{k}, \\ (\mathbf{0}, 0) & \text { in } \Omega \backslash \Omega_{k} .\end{cases}
$$

Note that, since $\beta>\frac{1}{2}$, the pair $(\mathbf{v}, q)$ belongs to $Z(\Omega)$ and that $\mathbf{v}$ vanishes on $\partial \Omega_{k}$. Inserting this into (53) and using the Cauchy-Schwarz inequality yields

$$
\begin{aligned}
& \left\|\left(\mathcal{I}_{N}^{k} \mathbf{f}+v \nabla^{2} \mathbf{u}_{N}-\nabla p_{N}\right) d_{k}^{\beta / 2}\right\|_{L^{2}\left(\Omega_{k}\right)^{2}}^{2} \\
& \quad \leqslant\left(\left|\mathbf{u}-\mathbf{u}_{N}\right|_{H^{1}\left(\Omega_{k}\right)^{2}}+\left\|p-p_{N}\right\|_{L^{2}\left(\Omega_{k}\right)}\right)|\mathbf{v}|_{H^{1}\left(\Omega_{k}\right)^{2}}+\left\|\mathbf{f}-\mathcal{I}_{N}^{k} \mathbf{f}\right\|_{L^{2}\left(\Omega_{k}\right)^{2}}\|\mathbf{v}\|_{L^{2}\left(\Omega_{k}\right)^{2}}
\end{aligned}
$$

Using (54), Lemmas 4 and 5 applied on $\widehat{\Omega}$ in both spatial directions and going back to the element $\Omega_{k}$, we get

$$
|\mathbf{v}|_{H^{1}\left(\Omega_{k}\right)^{2}}^{2} \leqslant c h_{k}^{4 \beta-2} N_{k}^{2(2-\beta)}\left\|\left(\mathcal{I}_{N}^{k} \mathbf{f}+v \nabla^{2} \mathbf{u}_{N}-\nabla p_{N}\right) d_{k}^{\beta / 2}\right\|_{L^{2}\left(\Omega_{k}\right)^{2}}^{2}
$$


Similarly, using (54), we have

$$
\|\mathbf{v}\|_{L^{2}\left(\Omega_{k}\right)^{2}}^{2} \leqslant h_{k}^{4 \beta}\left\|\left(\mathcal{I}_{N}^{k} \mathbf{f}+v \nabla^{2} \mathbf{u}_{N}-\nabla p_{N}\right) d_{k}^{\beta / 2}\right\|_{L^{2}\left(\Omega_{k}\right)^{2} .}^{2}
$$

Inserting all this in (58) yields

$$
\begin{aligned}
& \left\|\left(\mathcal{I}_{N}^{k} \mathbf{f}+v \nabla^{2} \mathbf{u}_{N}-\nabla p_{N}\right) d_{k}^{\beta / 2}\right\|_{L^{2}\left(\Omega_{k}\right)^{2}} \\
& \quad \leqslant c h_{k}^{2 \beta-1} N_{k}^{2-\beta}\left(\left|\mathbf{u}-\mathbf{u}_{N}\right|_{H^{1}\left(\Omega_{k}\right)^{2}}+\left\|p-p_{N}\right\|_{L^{2}\left(\Omega_{k}\right)}\right)+h_{k}^{2 \beta}\left\|\mathbf{f}-\mathcal{I}_{N}^{k} \mathbf{f}\right\|_{L^{2}\left(\Omega_{k}\right)^{2} .}
\end{aligned}
$$

Lemma 4 can now be used on the left-hand side of (61), so as to arrive at the result

$$
\begin{aligned}
& h_{k} N_{k}^{-1}\left\|\mathcal{I}_{N}^{k} \mathbf{f}+v \nabla^{2} \mathbf{u}_{N}-\nabla p_{N}\right\|_{L^{2}\left(\Omega_{k}\right)^{2}} \\
& \quad \leqslant c\left(N_{k}^{1+\beta}\left(\left|\mathbf{u}-\mathbf{u}_{N}\right|_{H^{1}\left(\Omega_{k}\right)^{2}}+\left\|p-p_{N}\right\|_{L^{2}\left(\Omega_{k}\right)}\right)+h_{k} N_{k}^{2 \beta-1}\left\|\mathbf{f}-\mathcal{I}_{N}^{k} \mathbf{f}\right\|_{L^{2}\left(\Omega_{k}\right)^{2}} .\right.
\end{aligned}
$$

So taking $\beta=\frac{1}{2}+\varepsilon$ for a small positive $\varepsilon$ leads to the desired estimate.

Bounding the second term in the definition (24) of $\eta^{k}$ is straightforward. Indeed, taking

$$
(\mathbf{v}, q)= \begin{cases}\left(\mathbf{0}, \boldsymbol{\nabla} \cdot \mathbf{u}_{N}\right) & \text { in } \Omega_{k}, \\ (\mathbf{0}, 0) & \text { in } \Omega \backslash \bar{\Omega}_{k},\end{cases}
$$

in (53) leads to

$$
\left\|\nabla \cdot \mathbf{u}_{N}\right\|_{L^{2}\left(\Omega_{k}\right)}^{2}=b\left(\mathbf{u}-\mathbf{u}_{N}, q\right) \leqslant\left|\mathbf{u}-\mathbf{u}_{N}\right|_{H^{1}\left(\Omega_{k}\right)^{2}}\|q\|_{L^{2}\left(\Omega_{k}\right)},
$$

whence

$$
\left\|\nabla \cdot \mathbf{u}_{N}\right\|_{L^{2}\left(\Omega_{k}\right)} \leqslant\left|\mathbf{u}-\mathbf{u}_{N}\right|_{H^{1}\left(\Omega_{k}\right)^{2}} .
$$

Finally, given some edge $\Gamma_{k, l}$ of an element $\Omega_{k}$, let $d_{k, l}$ denote the product of the distances from a point on $\Gamma_{k, l}$ to the end-points of the edge. Referring to the reference element $\widehat{\Omega}$ we may define a function $\widehat{\phi}$ which equals 1 on one edge of $\widehat{\Omega}$ and is zero on the opposite edge. Now suppose, for the sake of illustration, that an edge $\Gamma_{k, l}$ of the spectral element $\Omega_{k}$ is parallel to the $x$-axis, more precisely that it is contained in the line $y=c_{k}$. Then, for some $\beta, \frac{1}{2}<\beta<1$, we define on $\Omega_{k}$ the function

$$
\mathbf{v}_{k, l}(x, y)=\left[v \frac{\partial \mathbf{u}_{N}}{\partial n}-p_{N} \mathbf{n}\right]\left(x, c_{k}\right) d_{k, l}^{\beta}(x) \phi_{k, l}(y),
$$

where $\phi_{k, l}$ is a function derived from $\widehat{\phi}$ by translation and homothety, chosen equal to 1 on the edge $\Gamma_{k, l}$ and zero on the opposite edge of $\Omega_{k}$. Letting $\omega_{k, l}$ denote the union of elements the intersection of whose boundaries with $\Gamma_{k, l}$ has non-zero measure, our next choice of $(\mathbf{v}, q)$ is taken to be

$$
(\mathbf{v}, q)= \begin{cases}\left(\mathbf{v}_{k, l}, 0\right), & \text { in } \bar{\Omega}_{k}, \\ \left(\mathbf{v}_{k, l}^{r}, 0\right), & \text { in } \bar{\omega}_{k, l} \backslash \Omega_{k}, \\ (\mathbf{0}, 0), & \text { in } \Omega \backslash \omega_{k, l},\end{cases}
$$


(a)

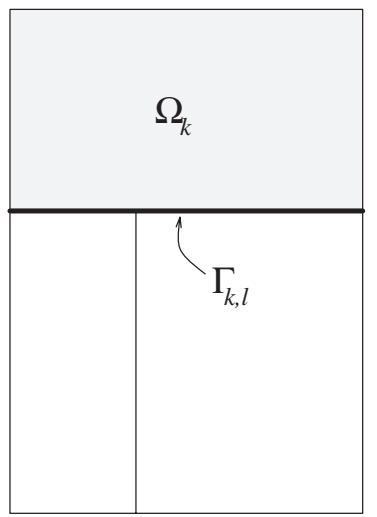

(b)

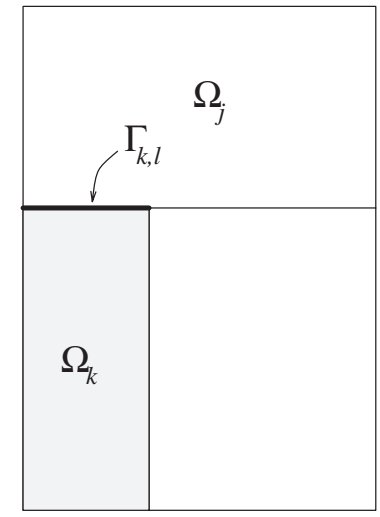

FIG. 1. $\Gamma_{k, l}$ is (a) the union of whole edges, (b) part of another edge.

where the choice of $\mathbf{v}_{k, l}^{r}$ depends on the geometry of the decomposition in the following ways:

(1) Suppose that $\Gamma_{k, l}$ is the union of whole edges of other elements as in Fig. 1 (a), for example. Then $\mathbf{v}_{k, l}^{r}$ is defined on these other elements by a scaled reflection along the interface: if $\Omega_{k}$ is the rectangle $] a_{k}, b_{k}[\times] c_{k}, d_{k}\left[, \mathbf{v}_{k, l}^{r}\right.$ is given by

$$
\mathbf{v}_{k, l}^{r}\left(x, c_{k}+\theta_{k}\left(c_{k}-y\right)\right)= \begin{cases}\mathbf{v}_{k, l}(x, y) & \text { for } a_{k} \leqslant x \leqslant b_{k}, c_{k} \leqslant y \leqslant d_{k} \\ \mathbf{0} & \text { elsewhere }\end{cases}
$$

where $\theta_{k}$ is positive and small enough for $] a_{k}, b_{k}[\times] c_{k}-\theta_{k}\left(d_{k}-c_{k}\right), c_{k}$ [ to be contained in $\omega_{k, l}$.

(2) Now suppose that $\Gamma_{k, l}$ contains a part of an edge of another element $\Omega_{j}$ (say) as in Fig. 1(b), for example. Then we write $\Omega_{j}=\Omega_{j 1} \cup \Omega_{j 2}$ where $\Omega_{j 1}$ is a rectangular subdomain of $\Omega_{j}$ which has $\Gamma_{k, l}$ as an entire edge. The function $\mathbf{v}$ is now defined in $\Omega_{j 1}$ by scaled reflection, as previously, and is set equal to zero in $\Omega_{j 2}$.

We insert this pair $(\mathbf{v}, q)$ into (53) and use the Cauchy-Schwarz inequality and thereby arrive at the inequality

$$
\begin{aligned}
\frac{1}{2}\left\|\left[v \frac{\partial \mathbf{u}_{N}}{\partial n}-p_{N} \mathbf{n}\right] d_{k, l}^{\beta / 2}\right\|_{L^{2}\left(\Gamma_{k, l}\right)^{2}}^{2} \\
\leqslant \\
\quad\left\|\left(\mathcal{I}_{N} \mathbf{f}+v \nabla^{2} \mathbf{u}_{N}-\nabla p_{N}\right) d_{k}^{\beta / 2}\right\|_{L^{2}\left(\omega_{k, l}\right)^{2}}\left\|\mathbf{v} d_{k}^{-\beta / 2}\right\|_{L^{2}\left(\omega_{k, l}\right)^{2}} \\
\quad+\left(\left|\mathbf{u}-\mathbf{u}_{N}\right|_{H^{1}\left(\left(\cup \Omega_{k}\right) ; \omega_{k, l}\right)^{2}}+\left\|p-p_{N}\right\|_{L^{2}\left(\omega_{k, l}\right)}\right)|\mathbf{v}|_{H^{1}\left(\left(\cup \Omega_{k}\right) ; \omega_{k, l}\right)^{2}} \\
\quad+\left\|\mathbf{f}-\mathcal{I}_{N} \mathbf{f}\right\|_{L^{2}\left(\omega_{k, l}\right)^{2}}\|\mathbf{v}\|_{L^{2}\left(\omega_{k, l}\right)^{2}}
\end{aligned}
$$

Let us now attempt to bound each of the norms appearing in (68). We begin by noting 
that

$$
\begin{aligned}
|\mathbf{v}|_{H^{1}\left(\left(\cup \Omega_{k}\right) ; \omega_{k, l}\right)^{2}}^{2} \leqslant & 3\left\|\frac{\partial}{\partial x}\left[v \frac{\partial \mathbf{u}_{N}}{\partial n}-p_{N} \mathbf{n}\right] d_{k, l}^{\beta} \phi_{k, l}\right\|_{L^{2}\left(\left(\cup \Omega_{k}\right) ; \omega_{k, l}\right)^{2}}^{2} \\
& +3 \beta^{2}\left\|\left[v \frac{\partial \mathbf{u}_{N}}{\partial n}-p_{N} \mathbf{n}\right] d_{k, l}^{\beta-1} d_{k, l}^{\prime} \phi_{k, l}\right\|_{L^{2}\left(\left(\cup \Omega_{k}\right) ; \omega_{k, l}\right)^{2}}^{2} \\
& +3\left\|\left[v \frac{\partial \mathbf{u}_{N}}{\partial n}-p_{N} \mathbf{n}\right] d_{k, l}^{\beta} \phi_{k, l}^{\prime}\right\|_{L^{2}\left(\left(\cup \Omega_{k}\right) ; \omega_{k, l}\right)^{2}}^{2}
\end{aligned}
$$

We use Lemma 5 for the first term in the right-hand side of (69) to obtain

$$
\begin{aligned}
& \left\|\frac{\partial}{\partial x}\left[v \frac{\partial \mathbf{u}_{N}}{\partial n}-p_{N} \mathbf{n}\right] d_{k, l}^{\beta} \phi_{k, l}\right\|_{L^{2}\left(\left(\cup \Omega_{k}\right) ; \omega_{k, l}\right)^{2}}^{2} \\
& \quad \leqslant c h_{k+}^{2 \beta-1} N_{k+}^{2(2-\beta)}\left\|\left[v \frac{\partial \mathbf{u}_{N}}{\partial n}-p_{N} \mathbf{n}\right] d_{k, l}^{\beta / 2}\right\|_{L^{2}\left(\Gamma_{k, l}\right)^{2}}^{2}\|\widehat{\phi}\|_{L^{2}(-1,1)}^{2} .
\end{aligned}
$$

To bound the second term, we observe that $\left|d_{k, l}^{\prime}(x)\right| \leqslant h_{k}$ and, using Lemma 4 with $\beta>\frac{1}{2}$, we see that

$$
\begin{aligned}
& \left\|\left[v \frac{\partial \mathbf{u}_{N}}{\partial n}-p_{N} \mathbf{n}\right] d_{k, l}^{\beta-1} d_{k, l}^{\prime} \phi_{k, l}\right\|_{L^{2}\left(\left(\cup \Omega_{k}\right) ; \omega_{k, l}\right)^{2}}^{2} \\
& \leqslant c h_{k+}^{3}\left\|\left[v \frac{\partial \mathbf{u}_{N}}{\partial n}-p_{N} \mathbf{n}\right] d_{k, l}^{\beta-1}\right\|_{L^{2}\left(\Gamma_{k, l}\right)^{2}}^{2}\|\widehat{\phi}\|_{L^{2}(-1,1)}^{2} \\
& \leqslant c h_{k+}^{2 \beta-1} N_{k+}^{2(2-\beta)}\left\|\left[v \frac{\partial \mathbf{u}_{N}}{\partial n}-p_{N} \mathbf{n}\right] d_{k, l}^{\beta / 2}\right\|_{L^{2}\left(\Gamma_{k, l}\right)^{2}}^{2}\|\widehat{\phi}\|_{L^{2}(-1,1)}^{2} .
\end{aligned}
$$

The use of (54) in the last term of (69) enables us to deduce that

$$
\begin{aligned}
\left\|\left[v \frac{\partial \mathbf{u}_{N}}{\partial n}-p_{N} \mathbf{n}\right] d_{k, l}^{\beta} \phi_{k, l}^{\prime}\right\|_{L^{2}\left(\left(\cup \Omega_{k}\right) ; \omega_{k, l}\right)^{2}}^{2} \\
\quad \leqslant c h_{k+}^{2 \beta-1}\left\|\left[v \frac{\partial \mathbf{u}_{N}}{\partial n}-p_{N} \mathbf{n}\right] d_{k, l}^{\beta / 2}\right\|_{L^{2}\left(\Gamma_{k, l}\right)^{2}}^{2}\left\|\widehat{\phi}^{\prime}\right\|_{L^{2}(-1,1)}^{2} .
\end{aligned}
$$

We also have

$$
\begin{aligned}
\|\mathbf{v}\|_{L^{2}\left(\omega_{k, l}\right)^{2}}^{2} & =\sum_{\Omega_{k} \subset \omega_{k, l}} \int_{\Omega_{k}}\left[v \frac{\partial \mathbf{u}_{N}}{\partial n}-p_{N} \mathbf{n}\right]^{2}(x) d_{k, l}^{2 \beta}(x) \phi_{k, l}^{2}(y) \mathrm{d} x \mathrm{~d} y, \\
& \leqslant c h_{k+}^{2 \beta+1}\left\|\left[v \frac{\partial \mathbf{u}_{N}}{\partial n}-p_{N} \mathbf{n}\right] d_{k, l}^{\beta / 2}\right\|_{L^{2}\left(\Gamma_{k, l}\right)^{2}}^{2}\|\widehat{\phi}\|_{L^{2}(-1,1)}^{2} .
\end{aligned}
$$

Finally,

$$
\left\|\mathbf{v} d_{k}^{-\beta / 2}\right\|_{L^{2}\left(\omega_{k, l}\right)^{2}}^{2} \leqslant c h_{k}^{-2 \beta+1}\left\|\left[v \frac{\partial \mathbf{u}_{N}}{\partial n}-p_{N} \mathbf{n}\right] d_{k, l}^{\beta / 2}\right\|_{L^{2}\left(\Gamma_{k, l}\right)^{2}}^{2}\left\|\left(1-\zeta^{2}\right)^{-\beta / 2} \widehat{\phi}\right\|_{L^{2}(-1,1)}^{2} .
$$


Therefore, inserting (69) to (74) in (68) leads to

$$
\begin{aligned}
\sum_{l=1}^{L(k)} \|[ & \left.v \frac{\partial \mathbf{u}_{N}}{\partial n}-p_{N} \mathbf{n}\right] d_{k, l}^{\beta / 2} \|_{L^{2}\left(\Gamma_{k, l}\right)^{2}} \\
\leqslant & c\left(\left\|\left(\mathcal{I}_{N} \mathbf{f}+v \nabla^{2} \mathbf{u}_{N}-\nabla p_{N}\right) d_{k}^{\beta / 2}\right\|_{L^{2}\left(\omega_{k}\right)^{2}} h_{k}^{1 / 2-\beta}\left\|\left(1-\zeta^{2}\right)^{-\beta / 2} \widehat{\phi}\right\|_{L^{2}(-1,1)}\right. \\
& +\left(\left|\mathbf{u}-\mathbf{u}_{N}\right|_{H^{1}\left(\left(\cup \Omega_{k}\right) ; \omega_{k}\right)^{2}}+\left\|p-p_{N}\right\|_{L^{2}\left(\omega_{k}\right)}\right) \\
& \times h_{k+}^{\beta-1 / 2}\left(N_{k+}^{2-\beta}\|\widehat{\phi}\|_{L^{2}(-1,1)}+\left\|\widehat{\phi}^{\prime}\right\|_{L^{2}(-1,1)}\right) \\
& \left.+\left\|\mathbf{f}-\mathcal{I}_{N} \mathbf{f}\right\|_{L^{2}\left(\omega_{k}\right)^{2}} h_{k+}^{\beta+1 / 2}\|\widehat{\phi}\|_{L^{2}(-1,1)}\right),
\end{aligned}
$$

and since from (61) we know a bound for $\left\|\left(\mathcal{I}_{N} \mathbf{f}+v \nabla^{2} \mathbf{u}_{N}-\nabla p_{N}\right) d_{k}^{\beta / 2}\right\|_{L^{2}\left(\omega_{k, l}\right)^{2}}$ we can now write

$$
\begin{aligned}
\sum_{l=1}^{L(k)} \| & {\left[v \frac{\partial \mathbf{u}_{N}}{\partial n}-p_{N} \mathbf{n}\right] d_{k, l}^{\beta / 2} \|_{L^{2}\left(\Gamma_{k, l}\right)^{2}} } \\
\leqslant & c \mu_{k}^{\beta+1 / 2}\left(\left(\left|\mathbf{u}-\mathbf{u}_{N}\right|_{H^{1}\left(\left(\cup \Omega_{k}\right) ; \omega_{k}\right)^{2}}+\left\|p-p_{N}\right\|_{L^{2}\left(\omega_{k}\right)}\right)\right. \\
& \times h_{k}^{\beta-1 / 2}\left(N_{k+}^{2-\beta}\|\widehat{\phi}\|_{L^{2}(-1,1)}+\left\|\widehat{\phi}^{\prime}\right\|_{L^{2}(-1,1)}+N_{k+}^{2-\beta}\left\|\left(1-\zeta^{2}\right)^{-\beta / 2} \widehat{\phi}\right\|_{L^{2}(-1,1)}\right) \\
& \left.+\left\|\mathbf{f}-\mathcal{I}_{N} \mathbf{f}\right\|_{L^{2}\left(\omega_{k}\right)^{2}} h_{k}^{\beta+1 / 2}\left(\left\|\left(1-\zeta^{2}\right)^{-\beta / 2} \widehat{\phi}\right\|_{L^{2}(-1,1)}+\|\widehat{\phi}\|_{L^{2}(-1,1)}\right)\right)
\end{aligned}
$$

We may choose, following Bernardi (1996), a representative polynomial $\widehat{\phi}$ such that $\widehat{\phi}(1)=0$ and $\widehat{\phi}(-1)=1$, to be

$$
\widehat{\phi}(\zeta)=(-1)^{N_{k}}\left(\frac{L_{N_{k}+1}^{\prime}(\zeta)}{\left(N_{k}+1\right)\left(N_{k}+2\right)}-\frac{L_{N_{k}}^{\prime}(\zeta)}{N_{k}\left(N_{k}+1\right)}\right),
$$

where $L_{n}=J_{n}^{0,0}$ denotes the Legendre polynomial with degree $n$. With $\widehat{\phi}$ so chosen, and using the standard results (Bernardi \& Maday, 1996, Section 3) that

$$
\int_{-1}^{1} L_{N_{k}}^{\prime 2}(\zeta) \mathrm{d} \zeta=N_{k}\left(N_{k}+1\right)
$$

we then have

$$
\|\widehat{\phi}\|_{L^{2}(-1,1)} \leqslant c N_{k}^{-1} .
$$

Therefore, applying Lemma 5 to $\widehat{\phi}$, we immediately see that

$$
\left\|\widehat{\phi}^{\prime}\right\|_{L^{2}(-1,1)} \leqslant c N_{k} .
$$

Moreover, Lemma 4 allows us to deduce for $\beta<1$, that

$$
\left\|\left(1-\zeta^{2}\right)^{-\beta / 2} \widehat{\phi}\right\|_{L^{2}(-1,1)}<c N_{k}^{\beta}\|\widehat{\phi}\|_{L^{2}(-1,1)} \leqslant c N_{k}^{\beta-1}
$$


Thus, we derive

$$
\begin{aligned}
\sum_{l=1}^{L(k)}\left\|\left[v \frac{\partial \mathbf{u}_{N}}{\partial n}-p_{N} \mathbf{n}\right] d_{k, l}^{\beta / 2}\right\|_{L^{2}\left(\Gamma_{k, l}\right)^{2}} & \\
\quad & c \lambda_{k}^{2-\beta} \mu_{k}^{\beta+1 / 2}\left(h_{k}^{\beta-1 / 2} N_{k}\left(\left|\mathbf{u}-\mathbf{u}_{N}\right|_{H^{1}\left(\left(\cup \Omega_{k}\right) ; \omega_{k}\right)^{2}}+\left\|p-p_{N}\right\|_{L^{2}\left(\omega_{k}\right)}\right)\right. \\
& +h_{k}^{\beta+1 / 2} N_{k}^{\beta-1}\left\|\mathbf{f}-\mathcal{I}_{N} \mathbf{f}\right\|_{\left.L^{2}\left(\omega_{k}\right)^{2}\right)},
\end{aligned}
$$

and we can multiply throughout by $h_{k}^{1 / 2} N_{k}^{-1 / 2}$, use Lemma 4 once more and take, as previously, $\beta=\frac{1}{2}+\varepsilon$ in order to arrive at a bound for the third term on the right-hand side of (24).

We have thus derived a bound for all the terms in the right-hand side of (24), which yields Theorem 2 .

REMARK As a conclusion, the estimate of Theorem 2 is not optimal according to the criteria proposed in Bernardi et al. (0000); however this seems unavoidable. Indeed, the lack of optimality which behaves essentially like $N_{k}^{3 / 2}$, is the same as in Bernardi (1996) for a much simpler problem.

\section{Numerical results}

In this section we shall use the derived error indicator (24) to guide a simple adaptive strategy for the numerical solution for steady Stokes flow boundary value problems where, generalising the problem description (1)-(3), non-homogeneous Dirichlet boundary conditions

$$
\mathbf{u}=\mathbf{g} \text { on } \partial \Omega \text {, }
$$

will be permitted. Of course, if a twice differentiable vector function can be found whose trace on the boundary is equal to $\mathbf{g}$ then there is no difficulty in rewriting the more general problem in the form (1)-(3). Such is the case in the example considered in Section 4.2.

The design of mesh modification strategies is an active area of research and several algorithms for $h p$ adaptive refinement have appeared in the literature (see, for example, Ainsworth \& Senior, 1998; Mavriplis, 1994; Oden et al., 1992; Valenciano \& Owens, 2000a,b). Oden et al. (1992) described a three step mesh refinement strategy. First, the initial mesh details were specified and the problem solved. Then $h$-refinements were applied in order to obtain an intermediate error index and equidistributed global error. Finally, $p$-enrichments were carried out to obtain a target error index. The strategy was later applied to finite element solutions of the Navier-Stokes equations (Oden et al., 1995) and viscoelastic flow problems (Warichet \& Legat, 1996, 1997). Mavriplis (1994) used an adaptive strategy for spectral element methods, where the decision to refine by adding more elements or increasing the order of the polynomials was made on the basis of the decay rate of the expansion coefficients in a Legendre polynomial expansion of the solution. The method was applied to both the one-dimensional Burgers equation and the Navier-Stokes driven cavity problem, although in the latter case only results with $h$-refinement were shown. Ainsworth \& Senior (1998) and Valenciano \& Owens (2000a,b) independently 
developed very similar adaptive refinement strategies for linear elliptic boundary value problems. The decision as to whether to $h$-refine or $p$-enrich in any given element was related by the authors to the relative magnitudes of the regularity of the exact solution (which would have to be estimated in most cases) and the polynomial order of the solution approximation.

In this section we outline a new and very simple mesh modification strategy of general applicability within the finite element or spectral element context to steady elliptic boundary value problems such as that described by (1)-(3). The purpose is to supply further evidence (in addition to that in Azaïez et al. (1996) and Valenciano \& Owens (2000b)) of the usefulness of the type of indicator proposed in (24) for guiding adaptive strategies.

\subsection{An $h N$ adaptive strategy}

The strategy is presented in pseudo-code form in Table 1. Supposing that at any stage in the $h N$ adaptive process there are $K$ spectral elements, the total error indicator

$$
\eta=\left(\sum_{k=1}^{K} \eta_{k}^{2}\right)^{1 / 2}
$$

is guaranteed to be less than some prescibed tolerance $\eta^{\text {tgt }}$ (say) by requiring that

$$
\eta_{k}<\frac{\eta^{\text {tgt }}}{\sqrt{K}}
$$

for all $k$. Hence, once an initial domain decomposition of the flow domain $\Omega$ into $K_{0}$ (say) elements $\Omega_{k}$ has been made and the maximum allowable value $\eta^{\text {tgt }}$ of $\eta$ has been chosen, uniform $N$-enrichment is done until $\eta_{k}<\eta^{\text {tgt }} / \sqrt{K_{0}} \forall k$. The ensemble of elements is then partitioned into those destined for $h$-refinements and $N$-enrichments (less regular elements) or just $N$-enrichments (more regular elements), depending upon the regularity in the elements of the solution. What happens next (main loop) is a two step mesh modification. After an $h$-refinement (B) in the less regular elements (bringing the new tally of elements to $K_{i}$ ) an attempt is made to decrease the polynomial order in the more regular elements (subroutine LayerReduce (C)) whilst maintaining $\eta_{k}<\eta^{\text {tgt }} / \sqrt{K_{i}}$ everywhere. In the second step the polynomial order in the less regular elements is reduced in steps of unity (D) and each reduction is followed by an increase in the polynomial order elsewhere just sufficient to ensure that the target error is achieved in all the elements. The cheapest mesh in the sequence of meshes generated by following the two steps is then selected and the main loop is repeated until no cheaper mesh can be found.

The question arises, of course, of how the regularity of the solution may be determined. We present three possibilities from the literature:

1. For linear elliptic boundary value problems having analytic data and coefficients, the solution will be analytic everywhere except at point singularities, such as at corners (Bernardi \& Raugel, 1981). Therefore, a subset of those elements containing the strongest singularities may be labelled 'less regular' and the remaining elements 'more regular'. This is what is done in Valenciano \& Owens (2000b) and in Section 4.2 of this paper. 
TABLE 1 Pseudo-code for the adaptive strategy

\section{(A) Initialisation}

(a) Define $\eta^{\text {tgt }}$, choose an initial decomposition into $K_{0}$ elements

(b) Label the elements $\Omega_{k}$ where the solution is more regular $S_{1,1}$ and the remaining elements $S_{2,1}$

(c) Denoting the polynomial order of the velocity approximation in a subset $\mathcal{S}$ of elements $\Omega_{k}$ by $N(\mathcal{S})$, set $N\left(S_{1,1}\right)=N\left(S_{2,1}\right)=N_{0}$, where $N_{0}$ is the minimum integer such that, for all $k, \eta_{k}<\eta^{\text {tgt }} / \sqrt{K_{0}}$ (d) Set $i=0$. Store mesh details $\mathcal{M}_{0}$

Main loop

$i=i+1$

(B) $h$-refine in $S_{i+1, i}$. There are now $K_{i}$ elements.

Define $S_{i+1, i+1}$ to be the ensemble of elements of $S_{i+1, i}$

where the solution is regular and $S_{i+2, i+1}=S_{i+1, i}-S_{i+1, i+1}$

Let $S_{k, i+1}=S_{k, i} k=1, \ldots, i$

(C) Call subroutine LayerReduce

Compute the number of degrees of freedom ndof 1

and store mesh details $\mathcal{M}_{i}$

(D) Let $N\left(S_{i+2, i+1}\right)=N\left(S_{i+2, i+1}\right)-1$

Let $N\left(S_{k, i+1}\right)=N\left(S_{k, i+1}\right)+N_{1}, \quad \forall k<i+2$,

where $N_{1}$ is the minimum integer such that $\eta_{k}<\eta^{\text {tgt }} / \sqrt{K_{i}} \quad \forall k$

Call subroutine LayerReduce

Compute the number of degrees of freedom ndof 2

If $n d o f 2<n d o f 1$ then

store mesh details $\mathcal{M}_{i}$ and repeat (D) end if

Goto start of main loop until $\operatorname{ndof}\left(\mathcal{M}_{i}\right)>\operatorname{ndof}\left(\mathcal{M}_{i-1}\right)$

stop

end

Subroutine LayerReduce

do $j=1, i+1$

$N\left(S_{j, i+1}\right)=N\left(S_{j, i+1}\right)-1$ while $\left(\left(\eta_{k}<\eta^{\mathrm{tgt}} / \sqrt{K_{i}} \forall k\right)\right.$.and. $\left(N\left(S_{j, i+1}\right)>N\left(S_{j-1, i+1}\right)\right.$ when $\left.\left.j>1\right)\right)$

end do

return

end 


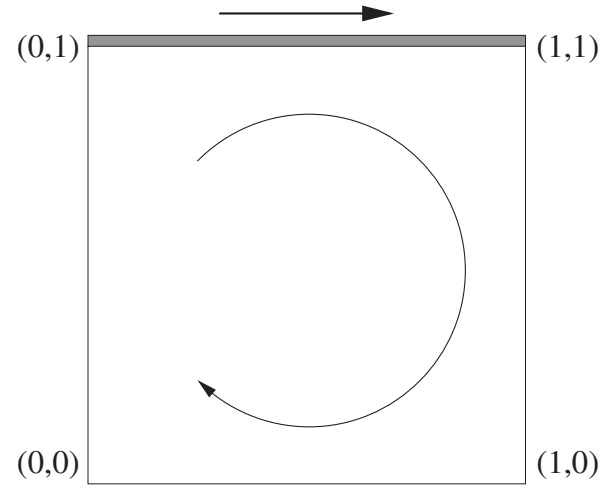

FIG. 2. The driven cavity geometry.

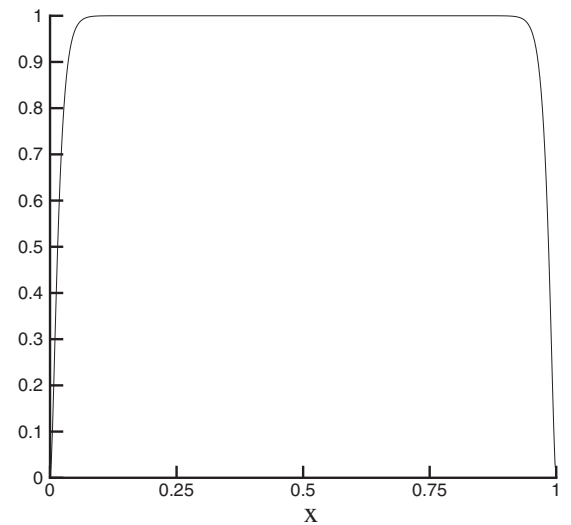

FIG. 3. Graph of the velocity profile $g(x)=\left(1-[2(x-0.5)]^{40}\right)^{2}$ on the upper plate $y=1$.

2. Ainsworth \& Senior (1998) estimate the solution error by solving local elemental boundary value problems with equilibration of the local fluxes. The local regularities then come out as a by-product of their a posteriori estimate.

3. Mavriplis (1994) estimates local regularities by calculating the decay rate of the expansion coefficients of the solution expressed in terms of a Legendre polynomial basis.

\subsection{An example}

As an example we will consider the steady Stokes regularised driven cavity problem (see Fig. 2), defined in a square $\Omega=[0,1]^{2}$. The 'lid' of the cavity $y=1$ moves horizontally with speed

$$
g(x)=\left[1-[2(x-0 \cdot 5)]^{40}\right]^{2} .
$$



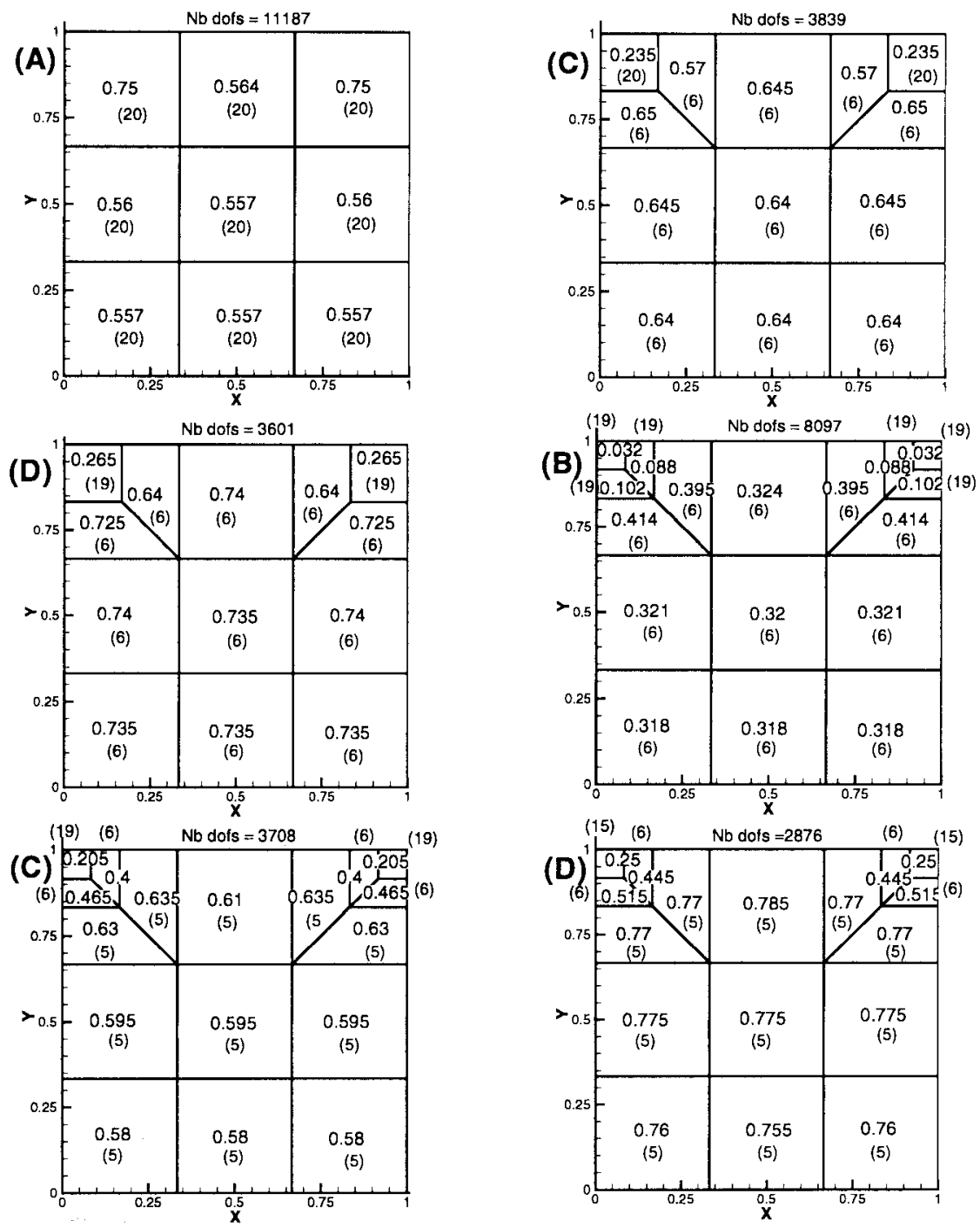

FIG. 4. Subsequence of meshes for case $\eta^{\text {tgt }}=0 \cdot 8$. The subsequence runs from left to right and top to bottom. The value of $\sqrt{K} \eta_{k}$ is noted in each element and the polynomial order $N$ is indicated in parentheses.

The lid velocity, shown in Fig. 3, is chosen so that although continuously differentiable at the corners $(0,1)$ and $(1,1)$, a steep layer has to be resolved in their proximity. A very similar velocity profile for the lid was used by Leriche (1999) and Leriche \& Gavrilakis (2000) in their computations using spectral methods for flow in a lid-driven cubical cavity. Although the solution $(\mathbf{u}, p)$ has singularities in all four corners of $\Omega$, the strength of the singularities in the lower two corners is not as great as in those at $y=1$. Indeed, in the 

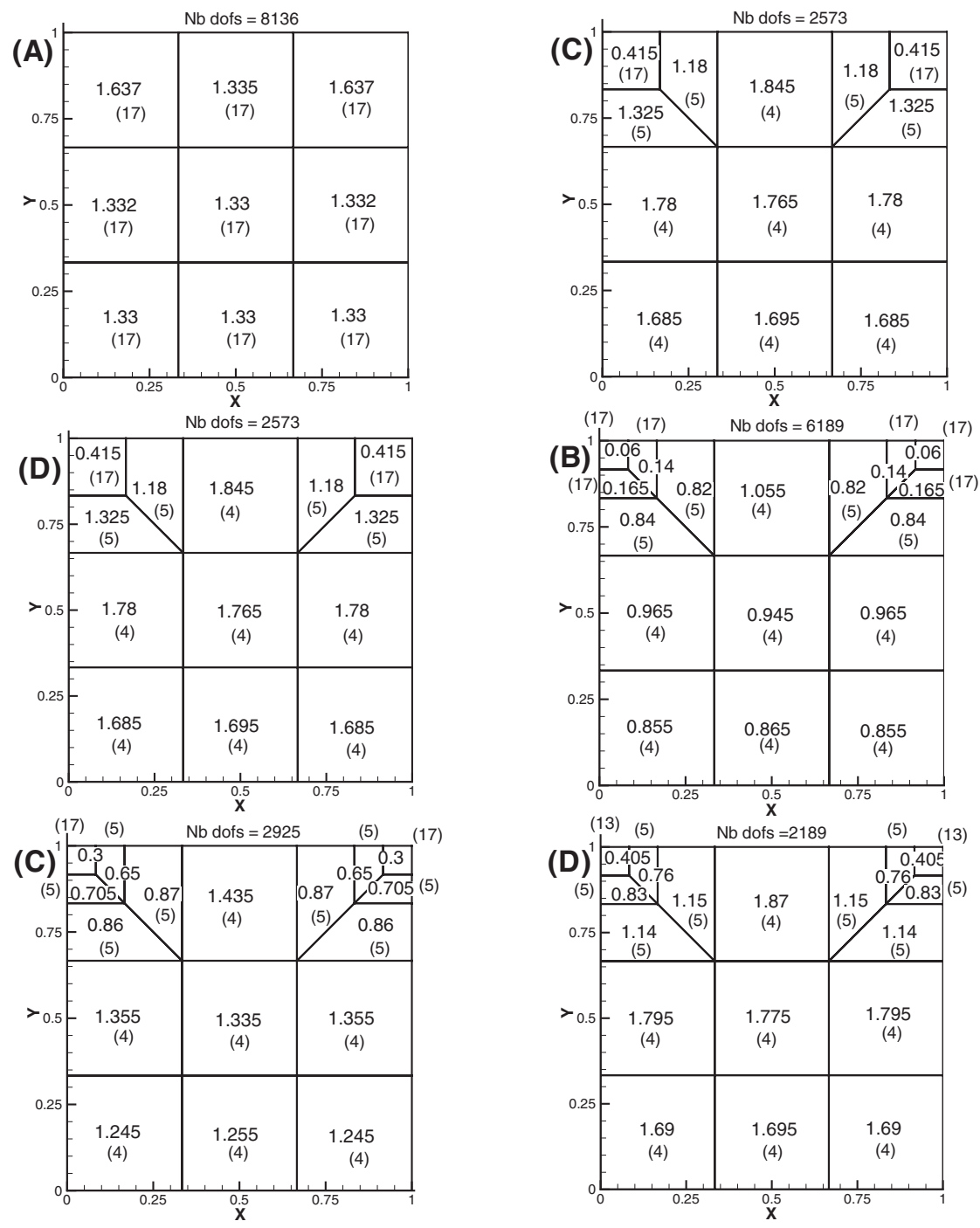

FIG. 5. Subsequence of meshes for case $\eta^{\text {tgt }}=1.9$. The subsequence runs from left to right and top to bottom. The value of $\sqrt{K} \eta_{k}$ is noted in each element and the polynomial order $N$ is indicated in parentheses.

elements $\Omega_{k}$ containing the lower two corners, the solution $(\mathbf{u}, p)$ belongs to $H^{3}\left(\Omega_{k}\right)^{2} \times$ $H^{2}\left(\Omega_{k}\right)$ (see (9)).

All numerical results shown in this paper were produced with SPECULOOS (Dubois-Pèlerin et al., 1999), a spectral element code developed in the Department of Mechanical Engineering at the Ecole Polytechnique Fédérale de Lausanne. The velocity approximations are based on Gauss-Lobatto-Legendre interpolating polynomials and the pressure approximation on Gauss interpolating polynomials of degree 2 less. Although the 


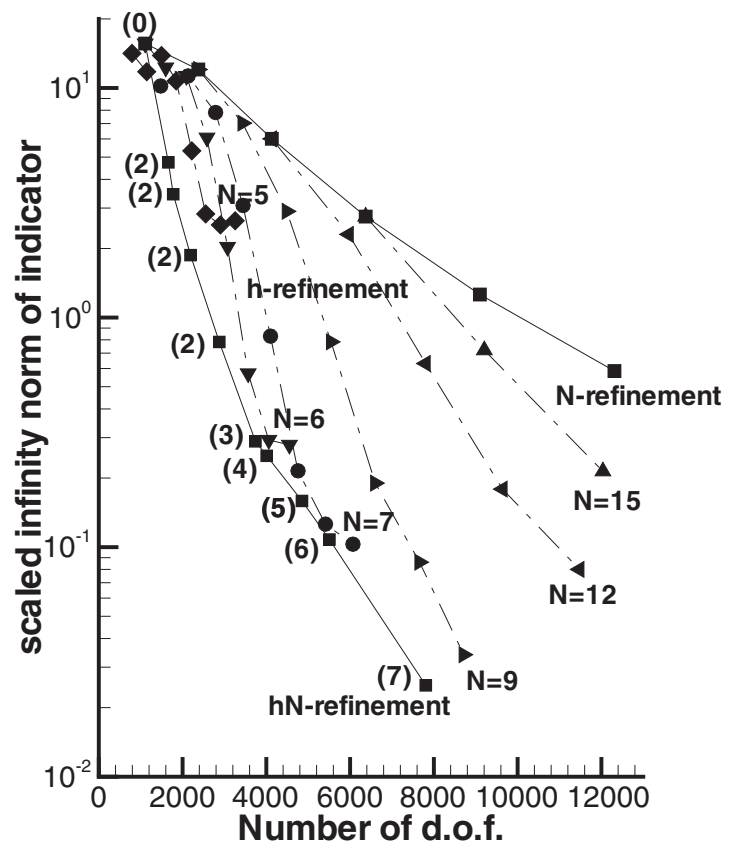

FIG. 6. Graph of total error bound $\sqrt{K} \eta_{k}^{\max }$ vs. degrees of freedom for $N$-refinements, $h$-refinements and the $h N$ adaptive strategy adopted in this paper. The number of passes through the main loop (see Table 1) required for each data point on the $h N$ curve are shown in parentheses.

code in its present form is unable to handle geometric non-conformities, flexibility in the choice of order of approximation from one element to another is ensured using the mortar method, as described in Section 2.2.

We choose, by way of illustrating the adaptive mesh refinement strategy detailed in Table 1, to do $h$-refinements and $N$-enrichments in the elements containing the upper corners and $N$-enrichments elsewhere. We make no claim about the optimality of the final mesh: the design of a sophisticated $h N$-adaptive strategy is outside the scope of the present paper.

The results of applying the strategy for $\eta^{\text {tgt }}=0.8$ and 1.9 are shown in Figs 4 and 5 respectively. In both cases, after the initialisation (stage A), the results of stages $\mathrm{C}$ and $\mathrm{D}(i=1)$ are shown, followed by the results of stages $\mathrm{B}, \mathrm{C}$ and $\mathrm{D}(i=2)$. No further iterations in the main loop were necessary.

It may be seen in the figures that considerably fewer degrees of freedom are required to achieve the convergence criteria than with a pure spectral element method ( $N$-type enrichments only) and moreover that the steep layer and singularities in the top corners are well resolved.

Let $K$ denote the number of spectral elements in a computational mesh for the test problem and $\eta_{k}^{\max }$ the maximum $\eta_{k}$ for $1 \leqslant k \leqslant K$. In Fig. 6 we show on a log-normal scale a graph of the total error bound $\sqrt{K} \eta_{k}^{\max }$ against the number of degrees of freedom for pure $N$-enrichments (nine equal sized elements), pure $h$-refinements (in the upper corners) for 
various values of polynomial order $N$, and the $h N$ adaptive strategy. The optimal number of passes through the main loop (detailed in Table 1) is shown next to each data point on the $h N$ adaptive curve. It may be seen that because of the presence of the strong singularities in the upper corners of the flow domain, pure $h$-refinements lead to a cheaper reduction in the error than is possible with $N$-enrichments alone. However, the $h$-refinement curves may also show an upturn for sufficiently small total error bounds. The $h N$ adaptive strategy gives rise to the cheapest meshes for all error bounds chosen, although several passes through the main loop may be necessary as the error bound becomes smaller.

\section{Conclusions}

In this paper we have proved properties of the error indicator (24), first described in the spectral element context in Valenciano \& Owens (2000b). The error indicator has been shown to be useful as a guide for an adaptive strategy. Future work will seek to extend the results contained in this paper to the Stokes equations in a three-dimensional axisymmetric domain and also to the full set of Navier-Stokes equations.

\section{REFERENCES}

Ainsworth, M. \& Oden, J. T. 1997 A posteriori error estimates for the Stokes and Oseen equations. SIAM J. Numer. Anal., 34, 101-113.

Ainsworth, M. \& SEnior, B. 1998 An adaptive refinement strategy for $h p$-finite element computations. Appl. Numer. Math., 26, 165-178.

AZAÏEZ, M., BERNARDI, C. \& MADAY, Y. 1996 Some tools for adaptivity in the spectral element method. Proceedings of the Third International Conference On Spectral And High Order Methods, (A. V. Jlin \& R. scotteds). Houston J. Math. pp. 243-253.

BANK, R. E. \& Welfert, B. D. 1990 A posteriori error estimators for the Stokes equations: a comparison. Comput. Meth. Appl. Mech. Eng., 82, 323-340.

Belhachmi, Z. 1997 Nonconforming mortar element methods for the spectral discretization of two-dimensional fourth-order problems. SIAM J. Numer. Anal., 34, 1545-1573.

BELHACHMI, Z. \& BERNARDI, C. 1994 Resolution of fourth-order problems by the mortar element method. Comput. Meth. Appl. Mech. Eng., 116, 53-58.

Ben Belgacem, F., Bernardi, C., Chorfi, N. \& Maday, Y. 2000 Inf-sup conditions for the mortar spectral element discretization of the Stokes problem. Numer. Math., 85, 257-281.

Bernardi, C. 1996 Indicateurs d'erreur en $h-N$ version des éléments spectraux. Modél. Math. Anal. Num., 30, 1-38.

BERNARDI, C. \& HeCHT, F. Error indicators for the mortar finite element discretization of the Laplace equation. Math. Comput. To appear.

Bernardi, C. \& MAday, Y. 1991 Polynomial approximation of some singular functions. Appl. Anal.: Int. J., 42, 1-32.

BERNARDI, C. \& MADAY, Y. 1996 Spectral methods. Handbook of Numerical Analysis, Techniques of Scientific Computing (Part 2), vol. 5. (P. G. Ciarlet \& J.-L. Lions, eds), Amsterdam: NorthHolland.

BERNARDI, C. \& MADAY, Y. 1999 Uniform inf-sup conditions for the spectral discretization of the Stokes problem. Math. Models Meth. Appl. Sci., 9, 395-414.

Bernardi, C., MAdAy, Y. \& PATERA, A. T. 1994 A new nonconforming approach to domain decomposition: the mortar element method. Nonlinear Partial Differential Equations and 
their Applications, Collège de France Seminar, XI, (Pitman Research Notes in Mathematics Series). (H. Brezis \& J.-L. Lions. eds), Longman, London, New York: pp. 13-51.

Bernardi, C., Métivet, B. \& Verfürth, R. Analyse numérique d'indicateurs d'erreur. Maillage et adaptation. (P.-L. George. ed.), Hermès. To appear.

Bernardi, C. \& RaUgel, G. 1981 Méthodes d'éléments finis mixtes pour les équations de Stokes et de Navier-Stokes dans un polygone non convexe. Calcolo, XVIII, 255-291.

Bertoluzza, S. \& Perrier, V. The mortar element method in the wavelet context. Modél. Math. et Anal. Numér. To appear.

Bertoluzza, S. \& Perrier, V. 2001 Coupling wavelets and finite elements by the mortar method. CR Acad. Sci. Paris. 332, 845-855.

BREZZI, F. 1974 On the existence, uniqueness and approximation of saddle point problems arising from Lagrangian multipliers. RAIRO Anal. Numér., 8, 129-151.

CAI, X.-C., DRYJA, M. \& SARKIS, M. 1997 Overlapping non-matching grid mortar element methods for elliptic problems. Technical Report CU-CS-842-97, Department of Computer Science, University of Colorado at Boulder.

Dubois-Pèlerin, Y., Van Kemenade, V. \& Deville, M. O. 1999 An object-oriented toolbox for spectral element analysis. J. Sci. Comput., 14, 1-29.

GiRAUlt, V. \& RAVIART, P.-A. 1986 Finite Element Methods for Navier-Stokes Equations, Theory and Algorithms. Berlin: Springer.

LERICHE, E. 1999 Direct numerical simulation of a lid-driven cavity flow by a Chebyshev spectral method, PhD Thesis, Department of Mechanical Engineering, EPFL, Switzerland.

LERICHE, E. \& GAVRILAKIS, S. 2000 Direct numerical simulation of the flow in a lid-driven cubical cavity. Phys. Fluids, 12, 1363-1376.

Maday, Y., MaVRiPlis, C. \& PATERA, A. T. 1989 Nonconforming mortar element methods: Application to spectral discretizations. Domain Decomposition Methods. Proceedings of the Second International Symposium on Domain Decomposition Methods, Los Angeles, California, 1988. (T. F. Chan, R. Glowinski, J. Periaux \& O. B. Widlund, eds), Philadelphia: SIAM, pp. 392-418.

Maday, Y., PAtera, A. T. \& RønQuist, E. M. 1992 The $\mathbb{P}_{N}-\mathbb{P}_{N-2}$ method for the approximation of the Stokes problem. Publications du Laboratoire d'Analyse Numérique R92025. Université Pierre et Marie Curie.

MAVRIPLIS, C. 1994 Adaptive mesh strategies for the spectral element method. Comput. Meth. Appl. Mech. Eng., 116, 77-86.

Oden, J. T., PAtra, A. \& Feng, Y. 1992 An $h-p$ adaptive strategy. AMD Adaptive, Multilevel, and Hierarchical Computational Strategies, 157, 23-47.

ODEN, J. T., Wu, W. \& LEGAT, V. 1995 An $h p$ adaptive strategy for finite element approximations of the Navier-Stokes equations. Int. J. Numer. Meth. Fluids, 20, 831-851.

OsBorn, J. E. 1976 Regularity of solutions of the Stokes problem in a polygonal domain. Numerical Solution of Partial Differential Equations III, Synspade 1975. Academic Press, pp. 393-411.

Valenciano, J. \& Owens, R. G. 2000a A new modification strategy for numerical solutions to elliptic boundary value problems. Appl. Numer. Math., 32, 305-329.

VAlenciano, J. \& OWEns, R. G. 2000b An $h-p$ adaptive spectral element method for Stokes flow. Appl. Numer. Math., 33, 365-371.

VERFÜRTH, R. 1989 A posteriori error estimators for the Stokes equations. Numer. Math., 55, 309325.

VERFÜRTH, R. 1991 A posteriori error estimators for the Stokes equations. II. Nonconforming discretizations. Numer. Math., 60, 235-249. 
VERFÜRTH, R. 1994 A posteriori error estimation and adaptive mesh-refinement techniques. $J$. Comput. Appl. Math., 50, 67-83.

WARICHET, V. \& LEGAT, V. 1996 Adaptive hp-finite element viscoelastic flow calculations. Comput. Meth. Appl. Mech. Eng., 136, 93-110.

WARICHET, V. \& LEGAT, V. 1997 Adaptive high-order prediction of the drag correction factor for the upper-convected Maxwell fluid. J. Non-Newtonian Fluid Mech., 73, 95-114.

Wohlmuth, B. 1999 A residual based error estimator for mortar finite element discretizations. Numer. Math., 84, 143-171. 